\title{
Phenomenology of a Quark Mass Matrix from Six Dimensions and its implication for the Strong CP problem
}

\author{
P. Q. Hung*, M. Seco*, A. Soddu* \\ Dept. of Physics, University of Virginia, \\ 382 McCormick Road, P. O. Box 400714, Charlottesville, Virginia 22904-4714
}

(December 10, 2018)

\begin{abstract}
A model of quark mass matrices from six dimensions, which is nearly democatic in nature and which is previously constructed by two of us (PQH and MS), is studied in detail in this manuscript. We found that not only it fits all the six quark masses as well as the CKM matrix but also that there exists a region in the allowed parameter space of the model where the constraint on the parameter $\bar{\theta}$ of the Strong CP problem is satisfied. This region itself puts a constraint on the CKM parameters $\bar{\rho}$ and $\bar{\eta}$. As such, through our analysis, there appears to be a deep connection between Strong and Weak CP in this model.
\end{abstract}

12.15.Ff, 12.60.Cn, 13.20.Eb, 14.60.Pq, 14.60.St, 14.70.Pw

Typeset using REVTEX 


\section{INTRODUCTION}

The search for a plausible model of fermion masses is a continuing quest in particle physics. In particular, the quark sector is a fertile ground to test various models since it is there that one has the largest amount of information: quark masses, CKM angles, CP phase, and it is in this sector that most models turn their attention to.

Two of us have recently constructed a model of fermion masses [1] in which the mass matrix is almost of the pure phase form and is constructed out of four plus two extra compact spatial dimensions. As shown in [1], one extra compact spatial dimension was needed to give a democratic mass matrix and another one was needed to make its matrix elements complex. In [1], an almost pure phase mass matrix was found to take the form $M=g_{Y} v / \sqrt{2}\left(1-\varrho_{i j}\right) \exp \left(i \theta_{i j}\right)$ with $i, j=1,2,3, \varrho_{i j}<<1$, and $\theta_{i j}<<1$, although Ref. [1] contains a more general result. Our motivation for that work was based on an attempt to give a theoretical basis for the so-called pure phase mass matrix (PPMM) ansatz (similar to the previous form but with $\varrho_{i j}=0$ ) of Ref. [2,3] which, at the time of its construction, was quite successful in fitting the various angles and masses. In so doing, we arrived at a mass matrix which contains the pure phase form as a particular limit. As we shall see below, the general result of Ref. [1] allows us to be able to fit the latest determination of the CKM elements [4]. Along the way, as stipulated in Ref. [1], we found a special region, in the allowed parameter space that fits the CKM matrix, where the parameter $\bar{\theta}$ of the famous strong CP problem can be found to satisfy the experimental bound $\bar{\theta}<10^{-9}$. This result is somewhat surprising since it is not at all evident that solutions of our model that fit the mass spectrum and the CKM matrix could also give values of $\bar{\theta}$ below the experimental bound. This connection between weak and strong CP is certainly very intriguing and will be the subject of our focus at the end of the paper.

The organization of the paper will be as follows. We first briefly review the construction of a democratic mass matrix (DMM) in five dimensions. We then summarize the salient points of the model of Ref. [1]: its construction in six dimensions and the resulting quark mass matrices. Next we enumerate and describe the parameters used in the numerical analysis of the mass matrices which is carried out in the section that follows. There we will show the allowed region in our parameter space where solutions are found to fit both the mass spectrum and the CKM matrix. Finally, we discuss a subspace in the allowed region where the bound $\bar{\theta}<10^{-9}$ is obeyed. In particular, we present some thoughts on the possible physics which might be responsible for this behaviour.

\section{DEMOCRATIC MASS MATRICES FROM FIVE DIMENSIONS}

Before discussing the results of Ref. [1], let us first review how a democratic mass matrix (DMM) [5] arises in the case with one extra compact dimension. A DMM is a special case of an almost PPMM with $\varrho_{i j}=0, \theta_{i j}=0$, namely one in which all matrix elements are unity, apart from a common factor, and hence the name "democratic". As described in [1], in order to obtain a democratic mass matrix and to avoid unwanted FlavourChanging Neutral Current processes (FCNC), we imposed the following permutation sym-

metries on the Action: $S_{3}^{Q} \otimes S_{3}^{U^{c}} \otimes S_{3}^{D^{c}}$, with $Q \rightarrow S_{3}^{Q} Q, U^{c} \rightarrow S_{3}^{U^{c}} U^{c}$ and $D^{c} \rightarrow S_{3}^{D^{c}} D^{c}$. $Q\left(x^{\alpha}, y\right), U^{c}\left(x^{\alpha}, y\right), D^{c}\left(x^{\alpha}, y\right)$ are the five-dimensional Dirac fields whose left-handed zero 
modes are given respectively by $q\left(x^{\alpha}\right), u_{R}^{c}\left(x^{\alpha}\right)$, and $d_{R}^{c}\left(x^{\alpha}\right)$. (For convenience, left-handed fields are used throughout [1] and in this paper with the last two fields representing actually the two quark $S U(2)_{L}$ singlets.) The extra dimension is compactified on an $S_{1} / Z_{2}$ orbifold. The action which obeys this permutation symmetry is the sum of two terms, $S_{0}$ and $S_{Y u k}$, where

$$
\begin{aligned}
& S_{0}= \int d^{5} x \bar{Q}_{i}\left(i \not D_{5}+f \phi(y)\right) Q_{i}+\bar{U}_{i}^{c}\left(i \not D_{5}+f \phi(y)-m_{U}\right) U_{i}^{c} \\
&+\bar{D}_{i}^{c}\left(i \not D_{5}+f \phi(y)-m_{D}\right) D_{i}^{c} \\
& S_{Y u k}=\int d^{5} x k_{U} \sum_{i} Q_{i}^{T} C_{5} H \sum_{j} U_{j}^{c}+\int d^{5} x k_{D} \sum_{i} Q_{i}^{T} C_{5} \tilde{H} \sum_{j} D_{j}^{c}+\text { h.c. }
\end{aligned}
$$

In Eq. (1) $D_{5}$ is the covariant derivative. (The gauge fields are supposed to be uniformly spread along the fifth dimension $y$ inside the thick brane.) $\phi(y) \delta_{i j}$ is the vacuum expectation value VEV for the background scalar field $\Phi\left(x^{\alpha}, y\right)$. The attractive proposal of [7] to localize chiral zero modes at different points along the extra dimension $y$ was adopted in [1]. As a result, $m_{U}$ and $m_{D}$ are the five dimensional "masses" which determine the locations of $u_{R}^{c}\left(x^{\alpha}\right)$ and $d_{R}^{c}\left(x^{\alpha}\right)$ along $y$. (As pointed out in Ref. [1], in order to have an invariant "mass term" under the $Z_{2}$ symmetry, one has to require a "mass reversal" $m \rightarrow-m$. The behaviour of $m$ under $Z_{2}$ could come for example in a model where the "masses" are generated by the radiative corrections to the $\operatorname{VEV} \phi(y), \phi(y) \rightarrow \phi(y)+\delta \phi$ with $\delta \phi$ being independant of $y$. Because $\Phi\left(x^{\alpha}, y\right) \rightarrow-\Phi\left(x^{\alpha}, L_{5}-y\right)$ under $Z_{2}$ symmetry, at the same time $\delta \phi \rightarrow-\delta \phi$ originating in this "mass reversal".) In Eq. (2) $k_{U}$ and $k_{D}$ are the Yukawa couplings in five dimensions which have been chosen real and flavor independant and $H\left(x^{\alpha}, y\right)$ is the five dimensional SM doublet Higgs field whose zero mode $h\left(x^{\alpha}\right)$ is assumed to be uniformly spread along $y$ inside the thick brane. Here $C_{5}=\gamma_{0} \gamma_{2} \gamma_{y}$ is the charge conjugation operator in five dimensions.

For the purpose of keeping track of the dimensionalities of various objects, let us remind ourselves that, in five dimensions, the Yukawa coupling $k_{U}$ has a (mass) dimension $M^{-1 / 2}$. A scalar field, in five dimensions, has a dimension $M^{3 / 2}$. The zero mode of the SM Higgs field can be written as $K h\left(x^{\alpha}\right)$ where $h\left(x^{\alpha}\right)$ is the usual 4-dimensional Higgs field with dimension $M$, and therefore the constant $C$ has a dimension $M^{1 / 2}$. In dimensionally reducing the above action to four dimensions, the following dimensionless combination appears in the Yukawa term:

$$
g_{Y, u} \equiv k_{U} K
$$

The effective action for the Yukawa term of the Up sector in four dimensions can now be written as

$$
S_{Y u k}^{e f f}=\int d^{4} x g_{Y, u} \sum_{i, j} q_{i}^{T}\left(x^{\alpha}\right) h\left(x^{\alpha}\right) C u_{j}^{c}\left(x^{\alpha}\right) \int d y \xi_{q}^{i}(y) \xi_{u^{c}}^{j}(y)+h . c . .
$$

where a similar expression holds for the Down sector. As stressed in Ref. [1], since all the $q_{i}$ 's are located at the same place inside the brane, and similarly for all the $u_{i}^{c}$ 's. The wave function overlap $\int d y \xi_{q}^{i}(y) \xi_{u^{c}}^{j}(y)$ is independant of $i, j$. The Yukawa action now becomes 


$$
S_{Y u k}^{e f f}=\int d^{4} x g_{Y, u}^{e f f} q^{T}\left(x^{\alpha}\right) h\left(x^{\alpha}\right)\left(\begin{array}{lll}
1 & 1 & 1 \\
1 & 1 & 1 \\
1 & 1 & 1
\end{array}\right) C u^{c}\left(x^{\alpha}\right)+h . c .
$$

where $g_{Y, u}^{e f f}$ is given by

$$
g_{Y, u}^{e f f}=g_{Y, u} \int d y \xi_{q}(y) \xi_{u^{c}}(y)
$$

and similarly for the down sector

$$
g_{Y, d}^{e f f}=g_{Y, d} \int d y \xi_{q}(y) \xi_{d^{c}}(y)
$$

From Eq. (5) one obtains the democratic mass matrix (DMM)

$$
M=g_{Y} \frac{v}{\sqrt{2}}\left(\begin{array}{lll}
1 & 1 & 1 \\
1 & 1 & 1 \\
1 & 1 & 1
\end{array}\right)
$$

which has eigenvalues $3 g_{Y} v / \sqrt{2}, 0$, 0, with $v \sim 246 G e V$. The DMM of Eq. (8) does not reproduce the right mass spectrum and the right CKM matrix. What has been shown in Ref. [1] is that by adding another compact extra dimension one can obtain a viable scenario represented by an almost-PPMM.

\section{ALMOST PURE PHASE MASS MATRICES FROM SIX DIMENSIONS}

\section{A. The Action}

The main idea of Ref. [1] is that by introducing a sixth compact extra dimension, and by requiring that the background scalar field couples to the fermions through a Yukawa interaction which is non-local along that extra dimension, one can obtain an oscillatory behavior for the fermion wave function along the sixth dimension. Fermions are delocalized along the sixth dimension, in contrast with the fifth dimension, and the oscillatory behavior

of the wave functions, together with the breaking of family symmetry, has the effect of producing phases in the mass matrix. Let us now summarize the main results obtained in Ref. [1]. We first rewrite more compactly the action given in Ref. [1] which is the sum of $S_{0}$ and $S_{Y u k}$ where

$$
\begin{aligned}
S_{0}= & \int d^{6} x\left[\bar{Q}_{i} i \not D_{6} Q_{i}+\bar{Q}_{i}(z)\left(\frac{f}{2} \phi_{i}(z)-\frac{m_{Q, i}}{2}\right) Q_{i}\left(L_{6}-z\right)-\bar{Q}_{i}\left(L_{6}-z\right)\left(\frac{f}{2} \phi_{i}(z)-\frac{m_{Q, i}}{2}\right) Q_{i}(z)\right. \\
& +\bar{U}_{i}^{c} i D_{6} U_{i}^{c}+\bar{U}_{i}^{c}(z)\left(\frac{f}{2} \phi_{i}(z)-\frac{m_{U, i}}{2}\right) U_{i}^{c}\left(L_{6}-z\right)-\bar{U}_{i}^{c}\left(L_{6}-z\right)\left(\frac{f}{2} \phi_{i}(z)-\frac{m_{U, i}}{2}\right) U_{i}^{c}(z) \\
& +\bar{D}_{i}^{c} i D_{6} D_{i}^{c}+\bar{D}_{i}^{c}(z)\left(\frac{f}{2} \phi_{i}(z)-\frac{m_{D, i}}{2}\right) D_{i}^{c}\left(L_{6}-z\right)-\bar{D}_{i}^{c}\left(L_{6}-z\right)\left(\frac{f}{2} \phi_{i}(z)-\frac{m_{D, i}}{2}\right) D_{i}^{c}(z) \\
& \left.+f^{\prime} \bar{Q}_{i} \Gamma_{7} \phi^{\prime}(y) Q_{i}+\bar{U}_{i}^{c} \Gamma_{7}\left(f^{\prime} \phi^{\prime}(y)-m_{U}\right) U_{i}^{c}+\bar{D}_{i}^{c} \Gamma_{7}\left(f^{\prime} \phi^{\prime}(y)-m_{D}\right) D_{i}^{c}\right]
\end{aligned}
$$

and 


$$
S_{Y u k}=\int d^{6} x k_{U} \sum_{i} Q_{i}^{T} C_{6} H \sum_{j} U_{j}^{c}+\int d^{6} x k_{D} \sum_{i} Q_{i}^{T} C_{6} \tilde{H} \sum_{j} D_{j}^{c}+\text { h.c. },
$$

where $C_{6}=\Gamma_{0} \Gamma_{2} \Gamma_{z}$ is the charge conjugation in six dimensions. (The gamma matrices in six dimensions can be obtained in Ref. [1].) In Eq. (9) we expressed the dependance from $z$ only for the non-local interaction terms. The important point here is that while these interactions will produce an oscillatory behavior for the fermion wave functions along the sixth dimensions, the local terms, which are built using $\Gamma_{7}$, are found to be responsible for localizing the fermions along the fifth dimension. The above actions are invariant under the family permutation symmetry $S_{3}^{Q} \otimes S_{3}^{U^{c}} \otimes S_{3}^{D^{c}}$.

The vacuum expectation values (VEV) for the background scalar fields $\Phi$ and $\Phi^{\prime}$ are given by

$$
<\Phi\left(x^{\alpha}, y, z\right)>=\left(\begin{array}{ccc}
\phi_{1}(z) & 0 & 0 \\
0 & \phi_{2}(z) & 0 \\
0 & 0 & \phi_{3}(z)
\end{array}\right)
$$

and

$$
<\Phi^{\prime}\left(x^{\alpha}, y, z\right)>=\left(\begin{array}{ccc}
\phi^{\prime}(y) & 0 & 0 \\
0 & \phi^{\prime}(y) & 0 \\
0 & 0 & \phi^{\prime}(y)
\end{array}\right)
$$

As in Ref. [1], the family symmetry is broken by the background scalar field $\phi_{i}(z) \delta_{i j}$ and by introducing different non-local "mass terms" $m_{Q, i}, m_{U, i}$ and $m_{D, i}$ for each family. To break the family symmetry together with the left right symmetry along the sixth dimension will allow us to reproduce the right mass spectrum and the right CKM matrix.

As shown in Ref. [1], the absence in Eq. (9) of local interactions of the form $\bar{\Psi} \Phi \Psi$, which will localize the fermions wave function along the sixth dimension, is obtained by introducing the discrete symmetry Q

$$
\begin{aligned}
\psi\left(x^{\alpha}, z\right) & \rightarrow Q \psi\left(x^{\alpha}, z\right)=\Gamma_{7} \psi\left(x^{\alpha}, z\right), \\
\psi\left(x^{\alpha}, L_{6}-z\right) & \rightarrow Q \psi\left(x^{\alpha}, L_{6}-z\right)=-\Gamma_{7} \psi\left(x^{\alpha}, L_{6}-z\right), \\
\Phi\left(x^{\alpha}, z\right) & \rightarrow Q \Phi\left(x^{\alpha}, z\right)=\Phi\left(x^{\alpha}, z\right) .
\end{aligned}
$$

As pointed out in Ref. [1] the realization of the Q-symmetry of Eq. (13) implies that the introduced orbifold for the compactification is $S_{1} /\left(Z_{2} \times Z_{2}^{\prime}\right)$ instead of $S_{1} / Z_{2}$. This also implies that the physical space is $\left[0, L_{6} / 2\right]$ instead of the initial support $\left[0, L_{6}\right]$. The nonlocal terms of Eq. (9) and the local terms containing $\Gamma_{7}$ are invariant under the above Q-symmetry.

\section{B. The Mass Matrix}

From the Yukawa action of Eq. (10) one can now obtain the effective action in four dimensions

$$
S_{Y u k}^{e f f}=\int d^{4} x g_{Y, u} \sum_{i, j} q_{i}^{T}\left(x^{\alpha}\right) h\left(x^{\alpha}\right) C u_{j}^{c}\left(x^{\alpha}\right) \int d y \xi_{5, q}^{i}(y) \xi_{5, u^{c}}^{j}(y) \int d z \xi_{6, q}^{i *}(z) \xi_{6, u^{c}}^{j}(z)+h . c . .
$$


where we considered only the up sector, but equal considerations hold for the down sector. Using Eqs. (5) and (6) one can rewrite $S_{Y u k}^{e f f}$ as

$$
S_{Y u k}^{e f f}=\int d^{4} x g_{Y, u}^{e f f} q^{T}\left(x^{\alpha}\right) h\left(x^{\alpha}\right)\left(\begin{array}{lll}
a_{11} & a_{12} & a_{13} \\
a_{21} & a_{22} & a_{23} \\
a_{31} & a_{32} & a_{33}
\end{array}\right) C u^{c}\left(x^{\alpha}\right)+h . c .,
$$

where

$$
\begin{aligned}
a_{i j}= & \int d z \xi_{6, q}^{i *}(z) \xi_{6, u^{c}}^{j}(z) \\
= & \frac{1}{L_{6}} \int_{0}^{L_{6}} d z \exp \left[-i\left(2 f V_{i} \ln \left(\cosh \left(\mu_{i} z\right)\right) / \mu_{i}-2 f V_{j} \ln \left(\cosh \left(\mu_{j} z\right)\right) / \mu_{j}\right.\right. \\
& \left.\left.-\left(m_{q, i}-m_{u, j}\right) z\right)\right]
\end{aligned}
$$

In Eq. (16) we have used for $\xi_{6, q}^{i}$ and $\xi_{6, u^{c}}^{i}$ respectively

$$
\begin{gathered}
\xi_{6, q}^{i}=\frac{1}{\sqrt{L_{6}}} \exp \left[i\left(2 f V_{i} \ln \left(\cosh \left(\mu_{i} z\right)\right) / \mu_{i}-m_{q, i} z\right)\right] \\
\xi_{6, u^{c}}^{i}=\frac{1}{\sqrt{L_{6}}} \exp \left[i\left(2 f V_{i} \ln \left(\cosh \left(\mu_{i} z\right)\right) / \mu_{i}-m_{u, i} z\right)\right]
\end{gathered}
$$

which correspond to a VEV $\phi_{i}(z)=V_{i} \operatorname{tgh}\left(\mu_{i} z\right)$ with $\mu_{i}=\sqrt{\lambda / 2} V_{i}$.

As pointed out in Ref. [1], $L_{6}$ is now a generic symbol for the length of the physical space, which is $L_{6} / 2$ for the orbifold $S_{1} /\left(Z_{2} \times Z_{2}^{\prime}\right)$. From Eq. (14) one obtains the mass matrix

$$
M=g_{Y, u}^{e f f} \frac{v}{\sqrt{2}}\left(\begin{array}{lll}
a_{11} & a_{12} & a_{13} \\
a_{21} & a_{22} & a_{23} \\
a_{31} & a_{32} & a_{33}
\end{array}\right)
$$

Following Ref. [1], if one now uses the linear approximation for the kink, which is valid for $1 / \mu_{i} \sim \mathcal{O}\left(L_{6}\right)$, all domain wall thiknesses along $z$ are of the size of the compact dimension, one can obtain for the elements $a_{i j}$ the form $\left(1-\varrho_{i j}\right) e^{i \theta_{i j}}$ with $\varrho_{i j} \ll 1$ and $\theta_{i j} \ll 1$. In the linear approximation for the kink one obtains the following expressions for $a_{i j}$

$$
a_{i j}=\frac{1}{L_{6}} \int_{0}^{L_{6}} d z \exp \left[-i\left(\Delta \mu_{i j}^{2} z^{2}-\Delta m_{i j}\right)\right]
$$

where

$$
\begin{gathered}
\Delta \mu_{i j}^{2} \equiv \frac{1}{2}\left(2 f V_{i} \mu_{i}-2 f V_{j} \mu_{j}\right), \\
\Delta m_{i j} \equiv m_{q, i}-m_{u, j} .
\end{gathered}
$$

As shown in Ref. [1], one can explicitly carry out the integration and obtain 


$$
a_{i j}=\frac{\sqrt{\pi}}{2} \frac{\operatorname{erf}\left(\frac{i\left(2 \Delta \mu_{i j}^{2} L_{6}-\Delta m_{i j}\right)}{2 \sqrt{i \Delta \mu_{i j}^{2}}}\right)+\operatorname{erf}\left(\frac{i \Delta m_{i j}}{2 \sqrt{i \Delta \mu_{i j}^{2}}}\right)}{\sqrt{i \Delta \mu_{i j}^{2}} L_{6}} \exp \left(i \frac{\left(\Delta m_{i j}\right)^{2}}{4 \Delta \mu_{i j}^{2}}\right) .
$$

Now if $\sqrt{\Delta \mu_{i j}^{2}} L_{6} \equiv x_{i} j \ll 1$ and $\Delta m_{i j} \equiv \ll 1$ one can expand Eq. (23) giving

$$
a_{i j}=\left(1-\frac{2}{45} x_{i j}^{4}-\frac{1}{24} y_{i j}^{2}+\frac{1}{12} x_{i j}^{2} y_{i j}\right) \exp \left(i\left(\frac{y_{i j}}{2}-\frac{x_{i j}^{2}}{3}\right)\right)+\mathcal{O}\left(x_{i j}^{8}, y_{i j}^{4}\right)
$$

which has the desired almost-PPMM form. It has to be stressed here that the expression for the mass matrix which has been used to make our numerical simulation is the one from Eq. (16). This implies that when we looked for a solution in the parameter space, we did not have to restrict ourselves to the particular range of values for the parameters where both the linear approximation for the kink and Eq. (24) were valid.

As pointed out in Ref. [1], by looking at Eqs. (19) and (16) one can make the important following consideration. If one introduces the same "mass" term for left and right components for each family $i$, which means that $m_{q, i}=m_{u, i}$ (and similarly $m_{q, i}=m_{d, i}$ for the Down sector), then the mass matrix of Eq. (19) is hermitian, i.e. $a_{i j}=a_{j i}^{*}$. In this particular case the mass matrices for the Up and Down sectors differ only by the Yukawa couplings and one will not be able to reproduce a realistic mass spectrum. Moreover the diagonalization matrices are the same, i.e. $V_{U}=V_{D}$, and $V_{C K M}=V_{U}^{\dagger} V_{D}$ becomes just the unit matrix. So in order to obtain a realistic mass spectrum and CKM matrix one needs to introduce different "mass" terms for left and right components at least for one sector. What will be shown in the following is that we will be able to reproduce the right mass spectrum and the right CKM matrix for the case in which both up and down mass matrices are not hermitian. While hermitian mass matrices do not give a realistic scenario, they have the important property of having a real determinant. This implies that the argument of their determinant is zero. As pointed out in [1], this fact could form the seed for a solution to the strong CP problem.

\section{DESCRIPTION OF THE PARAMETER SPACE}

In this section we are going to describe the parameter space for the model of Ref. [1]. The particular case we consider has 10 parameters. What has to be said here is that we started our numerical simulation considering cases with a higher number of parameters and only after examining the results obtained, we were able to reduce the parameter space to 10. This, by the way, is the same number of parameters found in the quark sector: Six masses and four CKM parameters. The 10 parameters we are considering are the following:

- $g_{Y, u}^{e f f}$ and $g_{Y, d}^{e f f}$ defined respectively by Eq. (6) and (7)

- $\mu_{1}, \mu_{2}$ and $\mu_{3}$ whose inverses give the thickness of the domain walls $\phi_{1}(z), \phi_{2}(z)$ and $\phi_{3}(z)$ of Eq. (11)

- $\varepsilon_{q, 1}=m_{q, 1} \sqrt{\lambda / 2} / 2 f$ and $\varepsilon_{q, 2}=m_{q, 2} \sqrt{\lambda / 2} / 2 f$ with $m_{q, 1}$ the "mass" term for the $1^{s t}$ family left component and $m_{q, 2}$ for the $2^{\text {nd }}$ and $3^{\text {rd }}$ family left components 
- $\varepsilon_{u, 2}=m_{u, 2} \sqrt{\lambda / 2} / 2 f$ and $\varepsilon_{u, 3}=m_{u, 3} \sqrt{\lambda / 2} / 2 f$ the "mass" term respectively for the $2^{n d}$ and $3^{\text {rd }}$ family right components, $\varepsilon_{u, 1}=0$

- $\Delta \varepsilon$ being the common split in $m \sqrt{\lambda / 2} / 2 f$ of $\varepsilon_{d, i}=\varepsilon_{u, i}-\Delta \varepsilon$ with respect to $\varepsilon_{u, i}$

As can be seen from the particular choice of parameters the background scalar potential does not break the left-right symmetry $\phi_{i}^{q}=\phi_{i}^{u}=\phi_{i}^{d}$ but on the other hand breaks the family symmetry $\phi_{i} \neq \phi_{j}$. The left-right symmetry is broken by choosing $\varepsilon_{q, i} \neq \varepsilon_{u, d, i}$ and by choosing different $\varepsilon_{i}$ for different indices $i$ one breaks additionally the family symmetry. The choice $\varepsilon_{q, 3}=\varepsilon_{q, 2}$ comes from the analysis of the same model in the case of 11 parameters, where all the $\varepsilon_{q, i}$ were different, and which gave as a result $\varepsilon_{q, 3} \simeq \varepsilon_{q, 2}$.

\section{RESULTS FOR MASS MATRICES FROM SIX DIMENSIONS}

In this section we will present the results obtained for the parameter space and for the quantities of Table 1.

We should point out that each solution that we found corresponds to a point in the parameter space with all the fitted quantities of Table 1 being in the experimental range [4,8]. In Figs. 1,2 and 3 we give the masses of the 6 quarks in $G e V$ evaluated at the $M_{Z}$ scale for three different cases corresponding to three different ranges of $\arg (\operatorname{det} M)=$ $\arg \left(\operatorname{det} M_{u}\right)+\arg \left(\operatorname{det} M_{d}\right)$. It will be clear in the next section why it is interesting to look at the quantity $\arg (\operatorname{det} M)$ when we present a scenario for a possible solution to the Strong CP problem. We choose to evaluate the running masses $m_{q}(\mu)$ at the scale $\mu=M_{Z}$, because the CKM matrix parameters $V_{i j}^{C K M}$ are given at $\mu=M_{Z}$. This is a common approach for quark mass matrix phenomenology. See Ref. [9] for a review of the running masses and the renormalization group equation that describes the evolution of the running quark masses $m_{q}(\mu)$ with the scale $\mu$. The edges of each box in Figs. 1-3 give the uncertanties for the masses, which depend not only on the errors of the input parameters for the renormalization group (RG) equation, but also on the error of the parameter which governs the flow itself, i.e. the strong coupling $\alpha_{s}\left(M_{Z}\right)$.

In the following we present two numerical examples corresponding respectively to $\arg (\operatorname{det} M) \sim O(1)$ and $\arg (\operatorname{det} M)<2 \times 10^{-10}$. For each numerical examples we will also give the corresponding parameter space. Below we rewrite the expression for the mass matrix to make clear the role of each parameter.

$$
\begin{aligned}
M_{i j} & =g_{Y e f f} \frac{v}{\sqrt{2}} \int d z \xi_{6, q}^{i *}(z) \xi_{6, u^{c}}^{j}(z) \\
& =g_{Y e f f} \frac{v}{\sqrt{2}} \frac{1}{L_{6}} \exp \left(-i 2 f \sqrt{\frac{\lambda}{2}}\right) \int_{0}^{L_{6}} d z \exp \left[-i\left(\ln \left(\cosh \left(\mu_{i} z\right)\right)-\ln \left(\cosh \left(\mu_{j} z\right)\right)-\left(\varepsilon_{q, i}-\varepsilon_{u, j}\right) z\right)\right]
\end{aligned}
$$

where $\varepsilon_{q, u, i}=m_{q, u, i} \sqrt{\lambda / 2} / 2 f$ with $\mu_{i}=\sqrt{\lambda / 2} V_{i}$. In our numerical simulation we will ignore the phase factor $\exp \left(-i 2 f \sqrt{\frac{\lambda}{2}}\right)$ in Eq. 25, which is independent of the indices $i, j$. All results correspond to $L_{6}=1$. 
One word of caution is in order here. In comparing our results with the phenomenological extractions of the CKM matrix elements, we take into account the following points. 1) The magnitudes of $V_{u b}$ and $V_{c b}$ are obtained from tree-level decays and are to a very good approximation independent of contributions from new physics. 2) If one were to use the Unitarity Triangle parameters $\bar{\rho}$ and $\bar{\eta}$ for comparison, one has to assume that possible new physics contributions (through loop effects for example) conspire to bring the apex $(\bar{\rho}, \bar{\eta})$ of the Unitarity Triangle to within the allowed band of the "unitarity clock". In the following we will use both points 1 and 2 to make our comparisons with experiments.

First, we give a numerical example corresponding to the case $\arg (\operatorname{det} M) \sim O(1)$. In Eq.s (26)-(30) we give the parameters space for the first case.

$$
\begin{gathered}
\mu_{1}=7.378, \quad \mu_{2}=8.460, \quad \mu_{3}=8.531, \\
\varepsilon_{q 1}=-8.262, \quad \varepsilon_{q 2}=5.090, \quad \varepsilon_{q 3}=5.090 \\
\varepsilon_{u 1}=0.000, \quad \varepsilon_{u 2}=1.120, \quad \varepsilon_{u 3}=1.198, \\
\varepsilon_{d 1}=-2.044, \quad \varepsilon_{d 2}=-0.924, \quad \varepsilon_{d 3}=-0.846, \\
g_{Y u} v / \sqrt{2}=152.31, \quad g_{Y d} v / \sqrt{2}=24.48 .
\end{gathered}
$$

We have decided to present the parameter space in a more readable way but it is important to remember that the number of independent parameters is 10. In Eqs. (31) and (33) we give the numerical expressions for the up and down quark mass matrix for the first case and in Eq.'s (32) and (34) the corresponding mass eigenvalues. As one can see, the elements of the mass matrices, although not equal to each other in magnitudes, are of the same order, revealing their democratic origins.

$$
\begin{gathered}
M_{u}=152.31 \mathrm{GeV}\left(\begin{array}{l}
0.1111+0.1690 i-0.1937-0.4167 i-0.1852-0.4377 i \\
0.1039+0.1750 i-0.1857-0.4221 i-0.1765-0.4430 i \\
0.1031+0.1758 i-0.1846-0.4238 i-0.1753-0.4446 i
\end{array}\right), \\
m_{u}=0.0023 \mathrm{GeV}, m_{c}=0.624 \mathrm{GeV}, m_{t}=183.0 \mathrm{GeV}, \\
M_{d}=24.48 \mathrm{GeV}\left(\begin{array}{l}
-0.0105+0.0003 i-0.0424-0.0086 i-0.0544-0.0121 i \\
-0.0090+0.0041 i-0.0442-0.0060 i-0.0563-0.0099 i \\
-0.0081+0.0043 i-0.0451-0.0060 i-0.0572-0.0100 i
\end{array}\right), \\
m_{d}=0.0048 \mathrm{GeV}, m_{s}=0.081 \mathrm{GeV}, m_{b}=3.09 \mathrm{GeV} .
\end{gathered}
$$

In Eqs. (35) and (36) we also give the absolute values of the mass matrices of Eqs. (31) and (33) which show that breaking the family symmetry does not destroy completely the democratic structure of the mass matrices. 


$$
\begin{gathered}
M_{u}=152.31 \mathrm{GeV}\left(\begin{array}{lll}
0.2023 & 0.4595 & 0.4753 \\
0.2035 & 0.4611 & 0.4768 \\
0.2038 & 0.4622 & 0.4780
\end{array}\right), \\
M_{d}=24.48 \mathrm{GeV}\left(\begin{array}{lll}
0.0105 & 0.0432 & 0.0558 \\
0.0099 & 0.0446 & 0.0571 \\
0.0092 & 0.0455 & 0.0581
\end{array}\right) .
\end{gathered}
$$

In Eqs. (37) and (38) we give the absolute values of the mass matrices of Eqs. (31) and (33) with rescaled values of the matrix elements. Eqs. (37) and (38) show in a more explicit way that the deviations from a democratic mass matrix are of $O(1)$.

$$
\begin{gathered}
M_{u}=72.80 \mathrm{GeV}\left(\begin{array}{lll}
0.4232 & 0.9614 & 0.9943 \\
0.4257 & 0.9647 & 0.9976 \\
0.4264 & 0.9671 & 1.0000
\end{array}\right), \\
M_{d}=1.42 \mathrm{GeV}\left(\begin{array}{lll}
0.1807 & 0.7446 & 0.9601 \\
0.1702 & 0.7675 & 0.9836 \\
0.1584 & 0.7836 & 1.0000
\end{array}\right) .
\end{gathered}
$$

In Eq. (39) we give the CKM matrix corresponding to the mass matrices of Eqs. 31 and (33), in Eq. (40) its absolute value, and in Eqs. (41) and (42) the values for the parameters $\bar{\rho}$ and $\bar{\eta}$, and for $\sin 2 \beta$ and $\gamma$.

$$
\begin{gathered}
V_{C K M}=\left(\begin{array}{ccc}
0.9711-0.0884 i & 0.0930-0.2014 i & -0.0036+0.0010 i \\
-0.1185-0.1872 i & 0.9734-0.0392 i & -0.0144+0.0398 i \\
0.0095-0.0064 i & 0.0150+0.0380 i & 0.9986+0.0302 i
\end{array}\right), \\
V_{C K M}=\left(\begin{array}{ccc}
0.9751 & 0.2218 & 0.0038 \\
0.2215 & 0.9742 & 0.0423 \\
0.0423 & 0.0409 & 0.9991
\end{array}\right), \\
\bar{\rho}=0.18, \quad \bar{\eta}=0.35 . \\
\sin 2 \beta=0.72, \quad \gamma=63.2^{0} .
\end{gathered}
$$

with $\bar{\rho}$ and $\bar{\eta}$ being defined as

$$
\begin{aligned}
& \bar{\rho}=\operatorname{Re}\left(V_{u d} V_{u b}^{*} V_{c d}^{*} V_{c b}\right) /\left|V_{c d} V_{c b}^{*}\right|^{2}, \\
& \bar{\eta}=\operatorname{Im}\left(V_{u d} V_{u b}^{*} V_{c d}^{*} V_{c b}\right) /\left|V_{c d} V_{c b}^{*}\right|^{2} .
\end{aligned}
$$

and $\sin 2 \beta$ and $\gamma$ as 


$$
\begin{gathered}
\sin 2 \beta=\frac{2 \bar{\eta}(1-\bar{\rho})}{(1-\bar{\rho})^{2}+\bar{\eta}^{2}} \\
\gamma=90^{0}-\frac{90^{0}}{\pi} \sin ^{-1}\left(\frac{2 \bar{\rho} \bar{\eta}}{\bar{\rho}^{2}+\bar{\eta}^{2}}\right)
\end{gathered}
$$

In Eq. (47) we give the values for the $\arg \left(\operatorname{det} M_{u}\right), \arg \left(\operatorname{det} M_{d}\right)$ and for their sum.

$$
\arg \left(\operatorname{det} M_{u}\right)=-1.5692, \arg \left(\operatorname{det} M_{d}\right)=1.8643, \arg (\operatorname{det} M)=0.2951 \text {. }
$$

The following numerical results corresponding to a value of $\arg (\operatorname{det} M)<10^{-9}$ are presented in the same way as the above example.

$$
\begin{aligned}
& \mu_{1}=7.365, \quad \mu_{2}=8.456, \quad \mu_{3}=8.532, \\
& \varepsilon_{q 1}=-8.189, \quad \varepsilon_{q 2}=5.026, \quad \varepsilon_{q 3}=5.026, \\
& \varepsilon_{u 1}=0.000, \quad \varepsilon_{u 2}=1.105, \quad \varepsilon_{u 3}=1.188, \\
& \varepsilon_{d 1}=-2.274, \quad \varepsilon_{d 2}=-1.168, \quad \varepsilon_{d 3}=-1.085, \\
& g_{Y u} v / \sqrt{2}=144.63, \quad g_{Y d} v / \sqrt{2}=23.60 . \\
& M_{u}=144.63 G e V\left(\begin{array}{ccc}
0.1154+0.1622 i & -0.1846-0.4383 i & -0.1740-0.4608 i \\
0.1096+0.1662 i & -0.179-0.4367 i & -0.1683-0.4591 i \\
0.1090+0.1670 i & -0.1781-0.4382 i & -0.1671-0.4606 i
\end{array}\right), \\
& m_{u}=0.0028 \mathrm{GeV}, \quad m_{c}=0.621 \mathrm{GeV}, \quad m_{t}=177.9 \mathrm{GeV}, \\
& M_{d}=23.60 \mathrm{GeV}\left(\begin{array}{ccc}
-0.0609+0.0113 i & -0.0167-0.0046 i-0.0294-0.0063 i \\
-0.0630+0.01480 i & -0.0144-0.0006 i-0.0272-0.0025 i \\
-0.0622+0.0147 i & -0.0152-0.0005 i-0.0280-0.0024 i
\end{array}\right) \text {, } \\
& m_{d}=0.0047 \mathrm{GeV}, \quad m_{s}=0.105 \mathrm{GeV}, \quad m_{b}=2.9 \mathrm{GeV} . \\
& M_{u}=144.63 \mathrm{GeV}\left(\begin{array}{lll}
0.1990 & 0.4756 & 0.4925 \\
0.1991 & 0.4720 & 0.4889 \\
0.1994 & 0.4730 & 0.4899
\end{array}\right) \\
& M_{d}=23.60 \mathrm{GeV}\left(\begin{array}{lll}
0.0619 & 0.0173 & 0.0301 \\
0.0647 & 0.0144 & 0.0273 \\
0.0639 & 0.0152 & 0.0281
\end{array}\right)
\end{aligned}
$$




$$
\begin{aligned}
& M_{u}=71.23 \mathrm{GeV}\left(\begin{array}{ccc}
0.4042 & 0.9656 & 1.000 \\
0.4043 & 0.9583 & 0.9927 \\
0.4049 & 0.9604 & 0.9948
\end{array}\right) \\
& M_{d}=1.53 \mathrm{GeV}\left(\begin{array}{lll}
0.9568 & 0.2676 & 0.4655 \\
1.0000 & 0.2229 & 0.4223 \\
0.9873 & 0.2350 & 0.4346
\end{array}\right) \\
& V_{C K M}=\left(\begin{array}{ccc}
-0.9681+0.1152 i & -0.1707+0.1425 i & 0.0038+0.0011 i \\
-0.1879-0.1183 & 0.9741-0.01456 i & -0.0078+0.0384 i \\
0.0068-0.0096 i & 0.0082+0.0367 i & 0.9991+0.0126 i
\end{array}\right) \\
& V_{C K M}=\left(\begin{array}{lll}
0.9750 & 0.2223 & 0.0040 \\
0.2220 & 0.9743 & 0.0392 \\
0.0118 & 0.0376 & 0.9992
\end{array}\right) \\
& \bar{\rho}=0.31, \quad \bar{\eta}=0.32, \\
& \sin 2 \beta=0.77, \quad \gamma=46.3^{0}, \\
& \arg \left(\operatorname{det} M_{u}\right)=-1.55845421528, \arg \left(\operatorname{det} M_{d}\right)=1.55845421485, \arg (\operatorname{det} M)=4.3 \times 10^{-10} \text {. }
\end{aligned}
$$

In Figs. 4, 5 and 6 we give the absolute values of the CKM matrix elements for the three different cases corresponding to three different ranges of $\arg (\operatorname{det} M)$. The uncertainties for each element are given by the edges of the corresponding window. Fig. 8 shows the solutions for $\bar{\rho}$ and $\bar{\eta}$. The sharp edges for the solution patches are due to the constraints imposed on $\bar{\rho}$ and $\bar{\eta}$. Fig. 9 shows instead the solutions for $\sin 2 \beta$ and $\gamma$. It has to be said here that the solutions appear in patches because of the way the minimization process works. One obviously can not exclude other solution patches. For example, could we try to make the minimization process follow different paths by toying with the input parameters, temperature and number of iterations, (see appendix A), and by changing the initial conditions. 


\section{TABLES}

\begin{tabular}{ccc}
\hline \hline$x_{i}$ & $\left\langle x_{i}>\right.$ & $\left|x_{i}^{\text {max }}-x_{i}^{\text {min }}\right| / 2$ \\
\hline$m_{u}$ & $2.33 \times 10^{-3}$ & $0.45 \times 10^{-3}$ \\
$m_{c}$ & 0.685 & 0.061 \\
$m_{t}$ & 181 & 13 \\
$m_{d}$ & $4.69 \times 10^{-3}$ & $0.66 \times 10^{-3}$ \\
$m_{s}$ & 0.0934 & 0.0130 \\
$m_{b}$ & 3.00 & 0.11 \\
$m_{u} / m_{d}$ & 0.497 & 0.119 \\
$m_{s} / m_{d}$ & 19.9 & 3.9 \\
$\left|V_{u d}\right|$ & 0.97485 & 0.00075 \\
$\left|V_{u s}\right|$ & 0.2225 & 0.0035 \\
$\left|V_{u b}\right|$ & 0.00365 & 0.00115 \\
$\left|V_{c d}\right|$ & 0.2225 & 0.0035 \\
$\left|V_{c s}\right|$ & 0.9740 & 0.0008 \\
$\left|V_{c b}\right|$ & 0.041 & 0.003 \\
$\left|V_{t d}\right|$ & 0.009 & 0.005 \\
$\left|V_{t s}\right|$ & 0.0405 & 0.0035 \\
$\left|V_{t b}\right|$ & 0.99915 & 0.00015 \\
$\bar{\rho}$ & 0.22 & 0.10 \\
$\bar{\eta}$ & 0.35 & 0.05 \\
$\sin 2 \beta$ & 0.78 & 0.08 \\
$\gamma$ & $59^{0}$ & $13^{0}$ \\
\hline \hline TABLE & Cent & \\
\hline
\end{tabular}

TABLE I. Central values and uncertainties for the masses of the 6 quarks evaluated at $M_{Z}$, for the two ratios $m_{u} / m_{d}$ and $m_{s} / m_{d}$, for the absolute values of the CKM matrix elements, the CP parameteres $\bar{\rho}, \bar{\eta}, \sin 2 \beta$ and $\gamma$. 
The parameter space with the 10 parameters described in the previous section and which corresponds to the three different cases for the the three different ranges of $\arg (\operatorname{det} M)$, are given in Figs. 10, 11 and 12. It has to be said here that the solutions presented correspond to a two step procedure. First we find solutions by requiring $\mu_{1}, \mu_{2}$ and $\mu_{3}$ to be larger than unity, because one can use in the physical space $\left[0, L_{6}\right]$ the kink solution for $\phi_{i}(z)$ instead of the kink-antikink approximate solution $\operatorname{Vtgh}(\mu z) \operatorname{tgh}\left(\mu\left(L_{6}-z\right)\right)$ [6] (the kink-antikink solution has the property of vanishing at both orbifold fixed point $z=0$ and $z=L_{6}$ as required by the imposed boundary conditions to compactify on an $S_{1} / Z_{2}$ orbifold). Second, using initial conditions from the parameter space already found, we looked for solutions whic correspond to very small ranges of $\mu_{1}, \mu_{2}$ and $\mu_{3}$. The reason we did this is because we noticed that the parameters which were more relevant to fit the quantities of Table 1 were the "mass" terms of left and right components, i.e $\varepsilon_{q, i}, \varepsilon_{u, i}$ and $\varepsilon_{d, i}$ respectively. As a result, in order to understand the dependence of the found solutions on the "mass" terms, we decided to restrict the range of $\mu_{1}, \mu_{2}$ and $\mu_{3}$. The way one controls a range for a parameter consists simply in adding that parameter to the quantities one wants to fit, modifying the function $f$ of Eq. (69) given in appendix A. For our numerical simulation we set $L_{6}=1$. This implies that the introduced parameters $\mu$ and $\varepsilon$ have to be multipied by $L_{6}$ for a general case.

We have presented in this section an analysis of the quark mass matrices obtained in Ref. [1]. In this analysis, we have used 10 parameters and were successful in fitting all 6 quark masses and all the parameters of the CKM matrix. This is shown in Figs. (1-10) for three separate ranges of the quantity $|\arg (\operatorname{det} M)|$ which was defined above. In these figures, each dot in the scatter plot represents a solution which fits the masses and the CKM matrix. As one can see, the solutions which correspond to $|\arg (\operatorname{det} M)| \sim 10^{-10}$ and which will have a significance to the Strong CP problem, are within $1 \sigma$ and $1.5 \sigma$ of the so-called R-fit and Bayesian fit respectively, as presented in Ref. [4].

\section{POSSIBLE CONNECTION BETWEEN STRONG AND WEAK CP}

In this section, we will discuss a possible connection between the region of parameter space where $\arg (\operatorname{det} M)<10^{-10}$ and the Strong CP problem.

It is well-known that the non-trivial vacuum of QCD generates a $\mathrm{P}$ and $\mathrm{CP}$ violating term in the Lagrangian of the form

$$
\mathcal{L}_{\bar{\theta}}=\frac{\bar{\theta}}{32 \pi^{2}} \tilde{G}_{\mu \nu} G^{\mu \nu}
$$

where

$$
\bar{\theta}=\theta_{Q C D}+\arg (\operatorname{det} M) .
$$

This term (66) gives a contribution to the electric dipole moment of the neutron [10], [11], with the current experimental limit [13] being $\bar{\theta}<2 \times 10^{-10}$. The mystery of why $\bar{\theta}$ should be so small constitutes the so-called Strong CP problem.

The most famous and elegant solution to the Strong CP problem is the Peccei-Quinn mechanism [14] where $\bar{\theta}$ becomes a dynamical field and relaxes to zero at the minimum of 
its potential. This dynamical field manifests itself as a pseudoscalar particle- the so-called Axion- whose decay constant is now severely constrained by astrophysical and cosmological arguments [15]. Another solution involving a massless up quark [16] is largely disfavoured by studies of chiral perturbation theory. A third type of solution to the Strong CP problem which has no axion, is the Nelson-Barr type of mechanism [17] which assumes exact CP at tree level and whose mass matrices have real determinants, and, as a result, the strong CP problem does not exist. It can arise at loop levels and can be "under control". However a realistic model of this type is yet to be constructed.

We have already mentioned that when we introduce the same "mass" term for left and right components (Eqs. (19) and (16)), i.e. $m_{q, i}=m_{u, i}=m_{d, i}$, the up and down quark mass matrices are hermitian and consequently their determinant is real [1]. $\arg \left(\operatorname{det} M_{u}\right)$ and $\arg \left(\operatorname{det} M_{d}\right)$ are separately zero. This situation suggests that the symmetry of the Lagrangian that makes $\arg (\operatorname{det} M)=0$ at tree level is the "left-right" symmetry of the components along the sixth dimension. If we also assume CP conservation at the Lagrangian level, this scenario would provide a solution to the strong CP problem. However this symmetry has to be broken because, as we have already mentioned, the case in which the quark mass matrices are hermitian does not reproduce the right mass spectrum and the right CKM matrix. One could imagine a scenario where the "left-right" symmetry is spontaneously broken. This will induce some loop corrections that will make the mass matrices deviate from hermiticity, reproducing the right mass spectrum and right CKM matrix. At the same time the breaking of this "left-right" symmetry could induce at loop levels a nonvanishing $\bar{\theta}$. But one can envision a scenario were the deviation from hermiticity happens in such a way that $\arg \left(\operatorname{det} M_{u}\right)$ and $\arg \left(\operatorname{det} M_{d}\right)$, each being now of $O(1)$, can cancel each other so as to keep $\bar{\theta}<2 \times 10^{-10}$.

As we have mentioned earlier, the solutions presented in this paper correspond to very small windows for the domain wall parameters $\mu_{i}$ 's, because we wanted to put in evidence the effect of the "left-right" symmetry breaking, especially in the $\bar{\theta}$ parameter. In searching for a quantity which could "retain the memory" of this "left-right" symmetry, we decided to plot, see Fig. 13, the sum of the arguments $|\arg (\operatorname{det}(M))|$ versus the "CoM", the weighted average of the "mass" terms along the sixth dimension, which is defined as

$$
C o M \equiv \frac{2 \sum_{i} \varepsilon_{q, i}+\sum_{i} \varepsilon_{u, i}+\sum_{i} \varepsilon_{d, i}}{12}
$$

As one can notice from Fig. 13, the sum of the arguments tends to go to zero for a particular $C o M \simeq 0.125$, and this behavior is confirmed in Fig. 14. Notice that, for practical reasons, we have reduced the number of points (i.e. the number of solutions) in Figs. 13 and 14 in order to reduce the sizes of the files containing these two figures. The actual number of solutions is much larger than what is shown in these figures.

Now one can think that the value of 0.125 for the "CoM" when the "left-right" symmetry is broken, and which corresponds to $\bar{\theta}<2 \times 10^{-10}$, was also the value for the "CoM" before the breaking, when $\bar{\theta}$ was equal to zero. In other words one can imagine a scenario where the "mass" terms of left and right components are split such a way as to retain the same value of the "CoM". To invent a mechanism which could break the "left-right" symmetry and which could reproduce the scenario mentioned above is beyond the scope of this paper, but it is one of the main topics we would like to investigate in the future. 
If one now looks at Fig. 8 which show the solutions in the $\bar{\rho}, \bar{\eta}$ plane for $|\arg (\operatorname{det} M)|$ in the three different ranges, one can see that the solutions corresponding to $10^{-12}<|\arg (\operatorname{det} M)|<$ $2 \times 10^{-10}$ tend to favor a particular region of the $\bar{\rho}, \bar{\eta}$ plane. In particular, the region of allowed solutions shrinks down in the $\bar{\rho}$ direction constraining the parameter $\bar{\rho}$ in the small window $\sim[0.3,0.32]$. These solutions are within $1 \sigma$ and $1.5 \sigma$ of the so-called R-fit and Bayesian fit respectively as can be seen from Fig. 8. This could be an artifact of the way the minimization process works, but it could also be an indication that there is a deep connection between Weak CP violation and Strong CP violation in our model.

As we have already mentioned earlier, one cannot exclude completely the existence of other solutions, because of the way the minimization process works, but we believe that the results obtained here can give some very good indications of how the allowed parameter space might look like.

\section{EPILOGUE}

We have presented in this paper a complete phenomenological analysis of the model of quark mass matrices as derived from six dimensions by [1]. With just 10 parameters, we have found a large number of solutions which can fit the 6 quark masses as well as the CKM matrix as can be seen in Figs. (1-9). Furthermore, we subdivide these solutions into three sets: 1) Those that have $\left.10^{-1}<|\arg (\operatorname{det} M)|<5 \times 10^{-1}, 2\right)$ those that have $10^{-3}<|\arg (\operatorname{det} M)|<10^{-1}$, and finally 3$)$ those that have $10^{-12}<|\arg (\operatorname{det} M)|<2 \times 10^{-10}$. The first two sets are given for the sole purpose of comparison with the last set which is most relevant to the Strong CP problem as discussed in the last section. As one can observe from Figs. (8) and (9), there is a deep connection between the weak CP parameters and the Strong

$\mathrm{CP}$ phase. In order to satisfy the constraint on the $\bar{\theta}$ parameter, i.e. $|\arg (\operatorname{det} M)|<2 \times 10^{-10}$ in our framework, the solutions obtained for $\bar{\rho}$ and $\bar{\eta}$ are found to be within $1 \sigma$ and $1.5 \sigma$ of the so-called R-fit and Bayesian fit respectively. As measurements of weak CP parameters become more accurate in the future, they will either rule out or confirm our predictions.

\section{ACKNOWLEDGMENTS}

We would like to thank Andrzej Buras and Gino Isidori for valuable comments. One of us (PQH) would like to thank the theory group of Laboratorio Nazionale di Frascati for hospitality during the course of this work. (AS) would like to thank Mike Timmins for introducing the simulated annealing method and Ngoc-Khanh Tran for usefull discussions. Our work is supported in parts by the US Department of Energy under grant No. DE-A50589ER40518.

\section{APPENDIX A}

As mentioned above the model we considered has 10 free parameters. Relying on this freedom we were able to fit the 6 quark masses evaluated at an energy scale equal to the mass of $\mathrm{Z}$ gauge boson $M_{Z}$, with constraints for the two ratios $m_{u} / m_{d}$ and $m_{s} / m_{d}$, the absolute values of the CKM matrix elements and the CP parameters $\bar{\rho}, \bar{\eta}$, or equivalently 
the three angles and one phase of the CKM matrix standard parametrization, for a total number of 10 quantities. The approach we used to derive the parameter space consists in minimizing a particular function, built in such a way that its global minima correspond to the region defined by the experimental constraints. This function is defined in the following way:

$$
\begin{aligned}
f & =\sum_{i=1}^{N} \frac{\left(x_{i}^{t h}-x_{i}^{\text {min }}\right)^{2}}{<x_{i}>^{2}} \theta\left(x_{i}^{\text {min }}-x_{i}^{\text {th }}\right) \\
& +\sum_{i=1}^{N} \frac{\left(x_{i}^{t h}-x_{i}^{\text {max }}\right)^{2}}{<x_{i}>^{2}} \theta\left(x_{i}^{\text {th }}-x_{i}^{\text {max }}\right)
\end{aligned}
$$

where $\theta(x)$ is the step function, $\mathrm{N}$ is the number of quantities that we want to fit, $x_{i}^{\text {th }}$ is the predicted value for the $i t h$ quantity, $x_{i}^{\min }$ and $x_{i}^{\max }$ fix the range for the ith quantity, and $\left\langle x_{i}\right\rangle$ is its average value. It is immediate to verify from Eq. (69) that when all the predicted quantities $x_{i}^{t h}$ 's are contained in the proper ranges, the function $f$ takes its minimum value equal to zero.

The set of parameters which correspond to a zero value for the function $f$ is called a solution. In our particular case the function we are considering is a mapping from $\mathcal{R}^{10}$ to $\mathcal{R}$. The parameter space is really big and to find the solutions, which correspond to the global minima of $f$, can be very challenging. The global minima of $f$ can be infact surrounded by a lot of local minima which most of the time can make the minimization process fail. Therefore, just trying different initial conditions with the hope to find the good one that will allow the minimization process to follow the right path towards a global minimum can result quite inefficient.

It is a common belief that there does not exist a general recipe to follow for minimization problems. A minimization procedure that can be very efficient for a particular problem, can be very inefficient in an another case or even fails. In the particular case where the function that we want to minimize depends on many parameters, there is a minimization procedure, called simulated annealing [18] [19], which seems to work more efficiently than others. This procedure is mostly used when the global minima are surrounded by a lot of local minima. Infact this minimization process can find a global minimum also after being trapped in a local minimum. The way instead most of the minimization processes work is to go, from the starting point, immediately downhill as far as they can go, but this often leads to a local minimum.

At the heart of the method of simulated annealing is an analogy with thermodynamics, specifically with the way liquids crystallize, or metal cool and anneal. When a liquid is cooled down sufficiently slowly, the atoms are often able to line themeselves up and form a pure crystal, which corresponds to the state of minimum energy for the system. But if the liquid is cooled quickly, it does not reach this state but rather ends up in an amorphous state of higher energy. So nature, as long as the process of cooling is sufficiently slow, is able to find the minimum energy state. The way nature works is based on the fact that for a system in thermal equilibrium at temperature $\mathrm{T}$, the probability for the system to be in a state of energy $E$ is given by the Boltzmann probability distribution:

$$
\operatorname{Prob}(E) \sim \exp \left(-\frac{E}{K T}\right) .
$$


This implies that even at low temperature there is a chance, albeit a tiny one, for the system to be in a high energy state. Therefore, there exists the possibility for the system to get out of a local energy minimum and move towards the global one. This principle has been incorporated in what is called a Metropolis algorithm [20]. Given a simulated thermodynamic system, it is assigned a probability $P=\exp \left(-\left(E_{2}-E_{1}\right) / K T\right)$ to the change from a configuration with energy $E_{1}$ to one with energy $E_{2}$. If $E_{2}<E_{1}, P$ is greater than unity and in this case to the change is assigned a probability $P=1$, which is equivalent to say that the system always makes such a change. In the case $E_{2}>E_{1}$, one can compare the probability $P=\exp \left(-\left(E_{2}-E_{1}\right) / K T\right)$ with a random-number and make the change to the new configuration only if $P>$ random - number. The system always takes a downhill step while sometimes takes an uphill step. The Metropolis algorithm can be used for systems other than the thermodynamic ones if we give:

- A description of possible system configurations.

- A generator of random changes in the configuration.

- A function $E$ (analog of energy) whose minimization is the goal of the procedure.

- A control parameter $T$ (analog of temperature) and an annealing schedule which tells how it is lowered, e.g. after how many random changes in configuration and with which step.

Going back to our particular case, the function $f$ we want to minimize is the analog of the energy, and each possible set of parameters correspond to a particular configuration of the system. For the algorithm to work a control parameter $T$, with an annealing schedule by which it is gradually reduced, has to be introduced, as well as a generator of random changes in the configuration, that is in the parameter space. The way these random changes are taken is the following: a positive quantity, given by $-T \cdot \ln ($ random - number $)$, is added to the stored function value. The same quantity is subtracted from the function value corresponding to every new set parameters that are tried as a replacement point in the parameter space. (The new points are obtained using the downhill simplex method. The simplex is a set of $N+1$ points with $\mathrm{N}$ the number of parameters, and the changes happen through reflections, expansions and contractions of the simplex). As mentioned before, this method allowed the system to jump from a local minimum and to look for a global one. The algorithm that we have used has been taken from numerical recipes [21]. Other than the initial set of values for the parameters, also the value for the temperature and the number of iterations which determine the annealing schedule to reduce the temperature, has to be given as input. The way these last two values were chosen, as well as the initials conditions is mostly the result of different attempts. The output of our code is a patch of solutions, which have been subsequently tested using an independent code written in Mathematica. 


\section{REFERENCES}

* e-mail:pqh@galileo.phys.virginia.edu

* e-mail: msm3v@galileo.phys.virginia.edu

* e-mail: asoddu@hep1.phys.ntu.edu.tw

[1] P. Q. Hung, M. Seco, Nucl. Phys. B 653, 123 (2003).

[2] G. C. Branco, J. I. Silva-Marcos and M. N. Rebelo, Phys. Lett. B 237446 (1990); G. C. Branco and J. I. Silva-Marcos, Phys. Lett. B 359, 166 (1995); G. C. Branco, D. Emmanuel-Costa and J. I. Silva-Marcos, Phys. Rev. D 56, 107 (1997).

[3] P. M. Fishbane and P. Q. Hung, Phys. Rev. D 57, 2743 (1998).

[4] D. Abbaneo et al., hep-ph/0304132 (2003)

[5] See e.g. P. Kaus and S. Meshkov, Phys. Rev. D 421863 (1990), and references therein.

[6] H. Georgi, A. K. Grant and G. Hailu, Phys. Rev. D 63, 064027 (2001).

[7] N. Arkani-Hamed, M. Schmaltz, Phys. Rev. D 61, 033005 (2000).

[8] Particle Data Group Collaboration, K. Hagiwara et al., Phys. Rev. D 66, 010001 (2002).

[9] H. Fusaoka and Y. Koide, Phys. Rev. D 57, 3986 (1998).

[10] V. Baluni, Phys. Rev. D 19, 2227 (1975).

[11] R. J. Crewther, P. di Vecchia, G. Veneziano, and E. Witten, Phys. Lett. B 88, 123 (1979); ibid 91 B, 487 (1980).

[12] A. E. Nelson, Phys. Lett. B 136, 387 (1983); B 143, 165 (1984).

[13] Particle Data Group, R. M. Barnett, et al., Phys. Rev. D 54, 1 (1996).

[14] R. D. Peccei and H. R. Quinn, Phys. Rev. D 16, 1791 (1977).

[15] For a review of various theories and search for the axion, see Review of Particle Physics, European Physical Journal C 15, (2000).

[16] J. Gasser, H. Leutwyler, Phys. Rep. 87, 77 (1982); H. Leutwyler, Nucl. Phys. B 337, 108 (1990); Phys. Lett. B 374, 163 (1996); Phys. Lett. B 378, 313 (1996).

[17] A. Nelson, Phys. Lett. B 136, 387 (1984); S. M. Barr, Phys. Rev. Lett. 53, 329 (1984); Phys. Rev. D 30, 1805 (1984). See also P. H. Frampton, P. Q. Hung, Marc Sher, Phys. Rep. 330, 263 (2000).

[18] S. Kirkpatrick, C. D. Gelatt, M. P. Vecchi, Science 220, 671-680 (1983).

[19] S. Kirkpatrick, Journal of Statistical Physics 34, 975-986 (1984).

[20] N. Metropolis, A. Rosenbluth. M. Rosenbluth, A. Teller, E. Teller, Journal of Chemical Physics 21, 1087-1092 (1953).

[21] NUMERICAL RECIPES in C, SECOND EDITION, W. H. Press, S. A. Teukolosky, W. T. Vetterling, B. P. Flannery, CAMBRIDGE. 


\section{FIGURES}

FIG. 1. Solutions for the 6 quark masses corresponding to $10^{-1}<|\arg (\operatorname{det} M)|<5 \times 10^{-1}$. The masses in $\mathrm{GeV}$ are evaluated at the $M_{Z}$ scale. The range for each mass as given in Table 1 is defined by the edges of the corresponding window.

FIG. 2. Solutions for the 6 quark masses corresponding to $10^{-3}<|\arg (\operatorname{det} M)|<10^{-1}$. The masses in $G e V$ are evaluated at the $M_{Z}$ scale. The range for each mass as given in Table 1 is defined by the edges of the corresponding window.

FIG. 3. Solutions for the 6 quark masses corresponding to $10^{-12}<|\arg (\operatorname{det} M)|<2 \times 10^{-10}$. The masses in $\mathrm{GeV}$ are evaluated at the $M_{Z}$ scale. The range for each mass as given in Table 1 is defined by the edges of the corresponding window.

FIG. 4. Solutions for the absolute values of the CKM elements corresponding to $10^{-1}<|\arg (\operatorname{det} M)|<5 \times 10^{-1}$. The range for each element as given in Table 1 is defined by the edges of the corresponding window. The ranges delimeted by the dashed lines correspond to the new evaluations for $\left|V_{u s}\right|$ (region on the left of the dashed line), $\left|V_{u b}\right|$ and $V_{c b}$ as in Ref. 4.

FIG. 5. Solutions for the absolute values of the CKM elements corresponding to $10^{-3}<|\arg (\operatorname{det} M)|<10^{-1}$. The range for each element as given in Table 1 is defined by the edges of the corresponding window. The ranges delimeted by the dashed lines correspond to the new evaluations for $\left|V_{u s}\right|$ (region on the left of the dashed line), $\left|V_{u b}\right|$ and $V_{c b}$ as in Ref. 4.

FIG. 6. Solutions for the absolute values of the CKM elements corresponding to $10^{-12}<|\arg (\operatorname{det} M)|<2 \times 10^{-10}$. The range for each element as given in Table 1 is defined by the edges of the corresponding window. The ranges delimeted by the dashed lines correspond to the new evaluations for $\left|V_{u s}\right|$ (region on the left of the dashed line), $\left|V_{u b}\right|$ and $V_{c b}$ as in Ref. 4.

FIG. 7. Solutions for the ratio $\left|V_{u b}\right| /\left|V_{c b}\right|$ for the three cases corresponding to a) $10^{-1}<|\arg (\operatorname{det} M)|<5 \times 10^{-1}, \quad$ b) $10^{-3}<|\arg (\operatorname{det} M)|<10^{-1}$, and c) $10^{-12}<|\arg (\operatorname{det} M)|<2 \times 10^{-10}$.

FIG. 8. Solutions for $\bar{\rho}$ and $\bar{\eta}$ for the three cases, from top to bottom, corresponding to a) $10^{-1}<|\arg (\operatorname{det} M)|<5 \times 10^{-1}$, b) $10^{-3}<|\arg (\operatorname{det} M)|<10^{-1}$, and c) $10^{-12}<|\arg (\operatorname{det} M)|<2 \times 10^{-10}$. The delimeted area is the allowed region in the $\bar{\rho}, \bar{\eta}$ plane as obtained from Ref. 4.

FIG. 9. Solutions for $\sin 2 \beta$ and $\gamma$ for the three cases, from top to bottom, corresponding to a) $10^{-1}<|\arg (\operatorname{det} M)|<5 \times 10^{-1}$, b) $10^{-3}<|\arg (\operatorname{det} M)|<10^{-1}$, and c) $10^{-12}<|\arg (\operatorname{det} M)|<2 \times 10^{-10}$. 
FIG. 10. Summary of the 10 parameters space corresponding to $10^{-1}<|\arg (\operatorname{det} M)|<5 \times 10^{-1}$.

FIG. 11. Summary of the 10 parameters space corresponding to $10^{-3}<|\arg (\operatorname{det} M)|<10^{-1}$.

FIG. 12. Summary of the 10 parameters space corresponding to $10^{-12}<|\arg (\operatorname{det} M)|<$ $2 \times 10^{-10}$.

FIG. 13. Solutions for $|\arg (\operatorname{det} M)|$ in the region $\left[10^{-3}, 5 \times 10^{-1}\right]$ vs the CoM as defined by Eq. 68.

FIG. 14. Solutions for $|\arg (\operatorname{det} M)|$ in the region $\left[10^{-12}, 2 \times 10^{-10}\right]$ vs the CoM as defined by Eq. 68. 

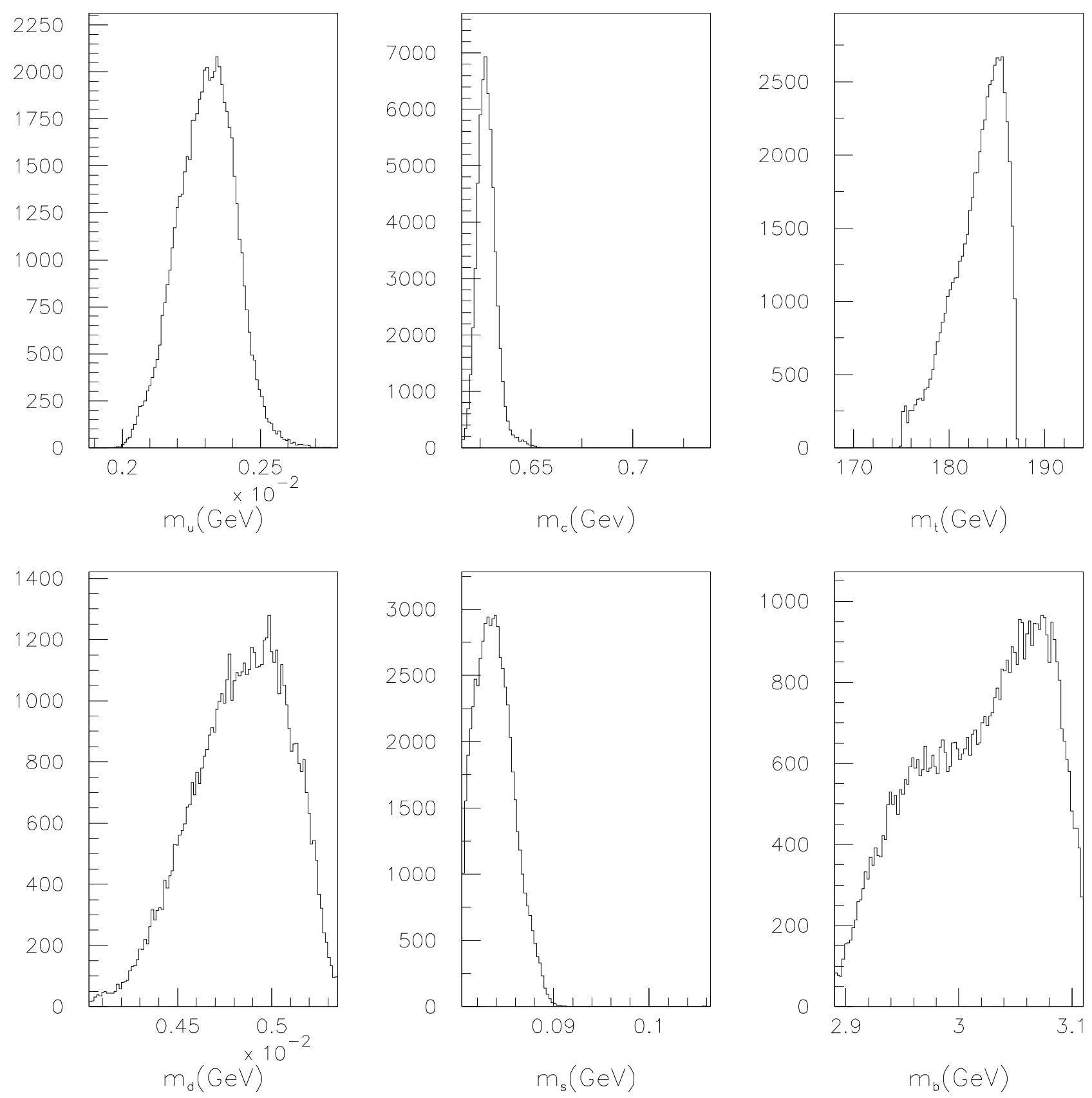

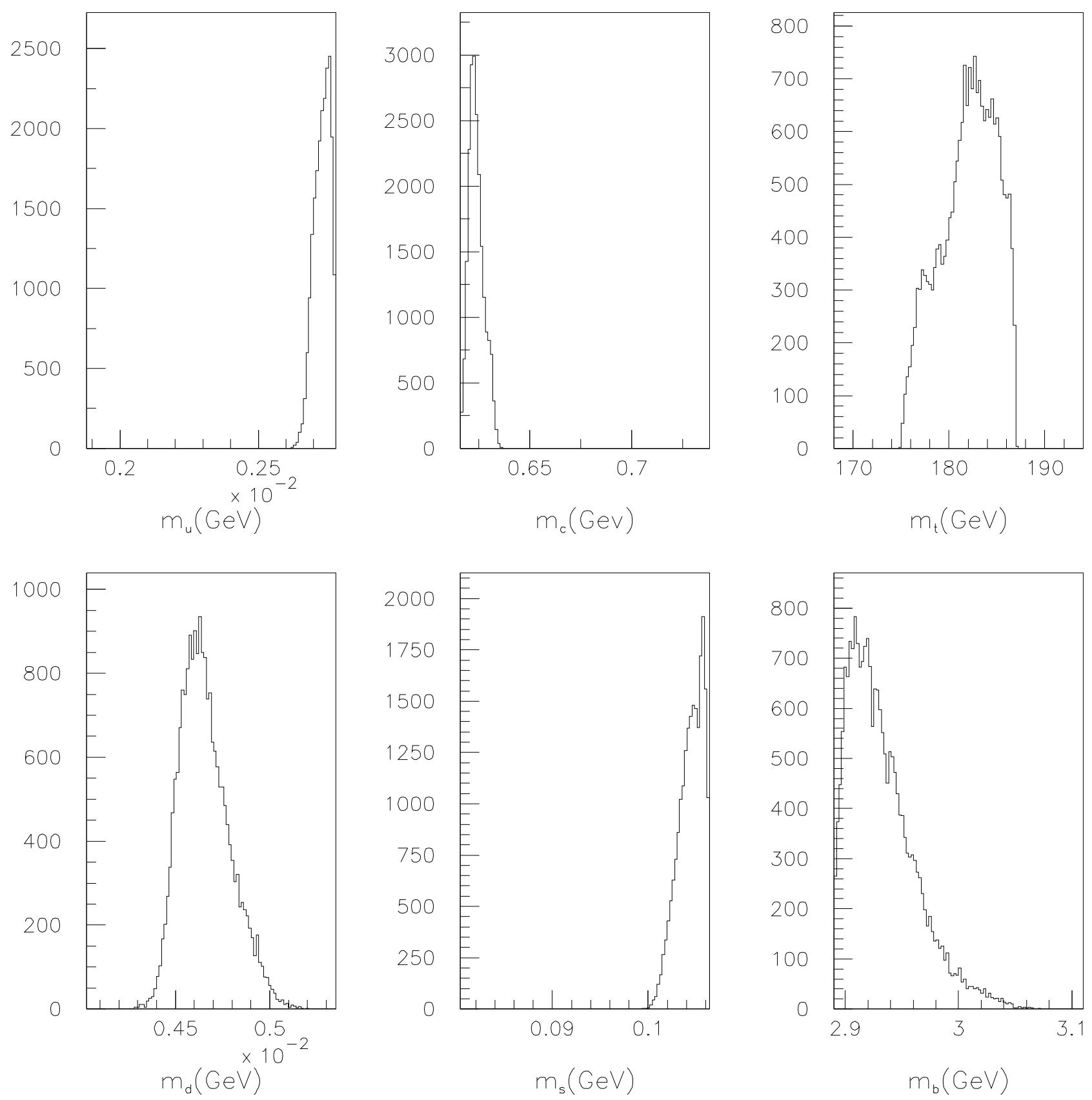

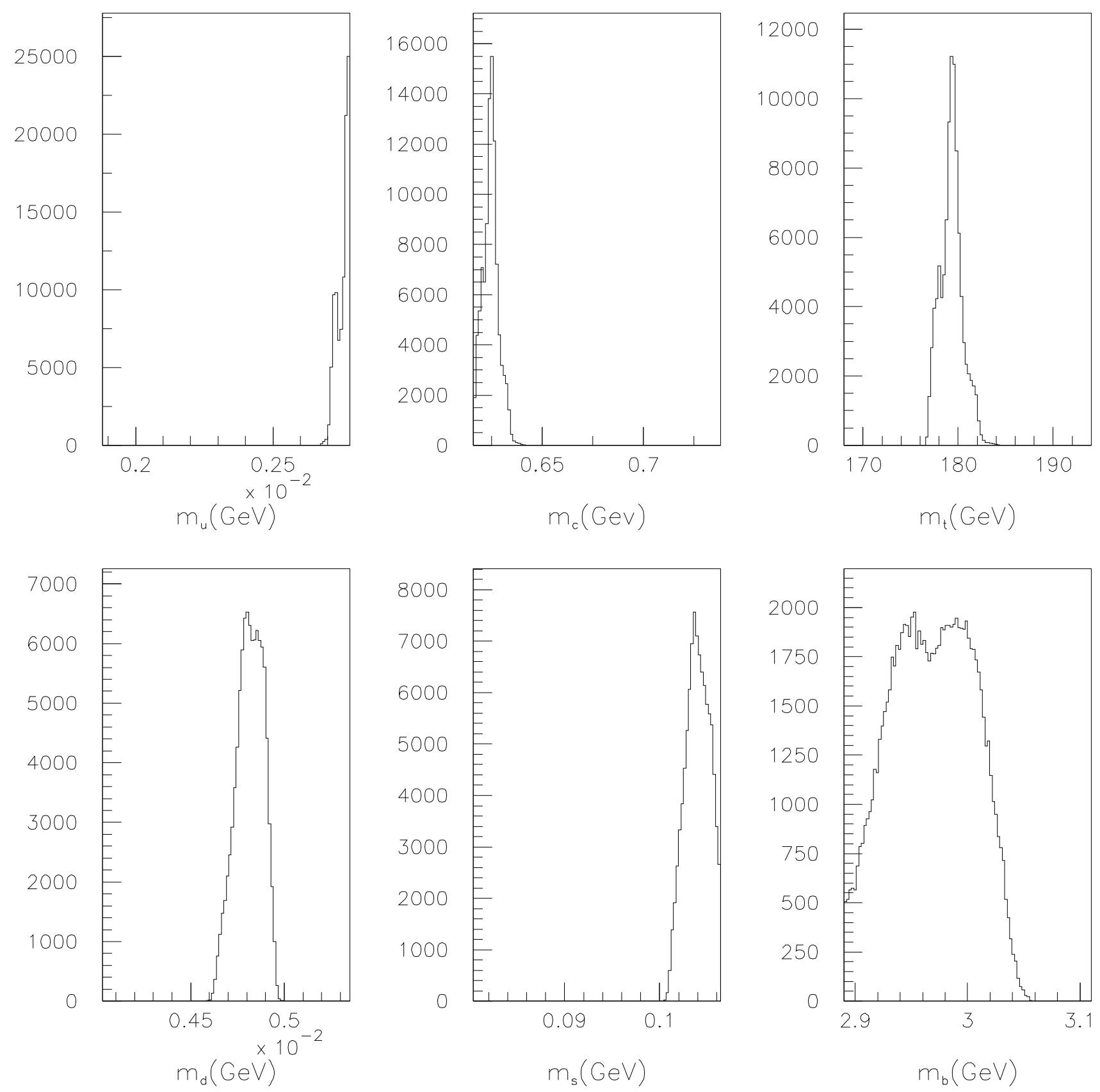

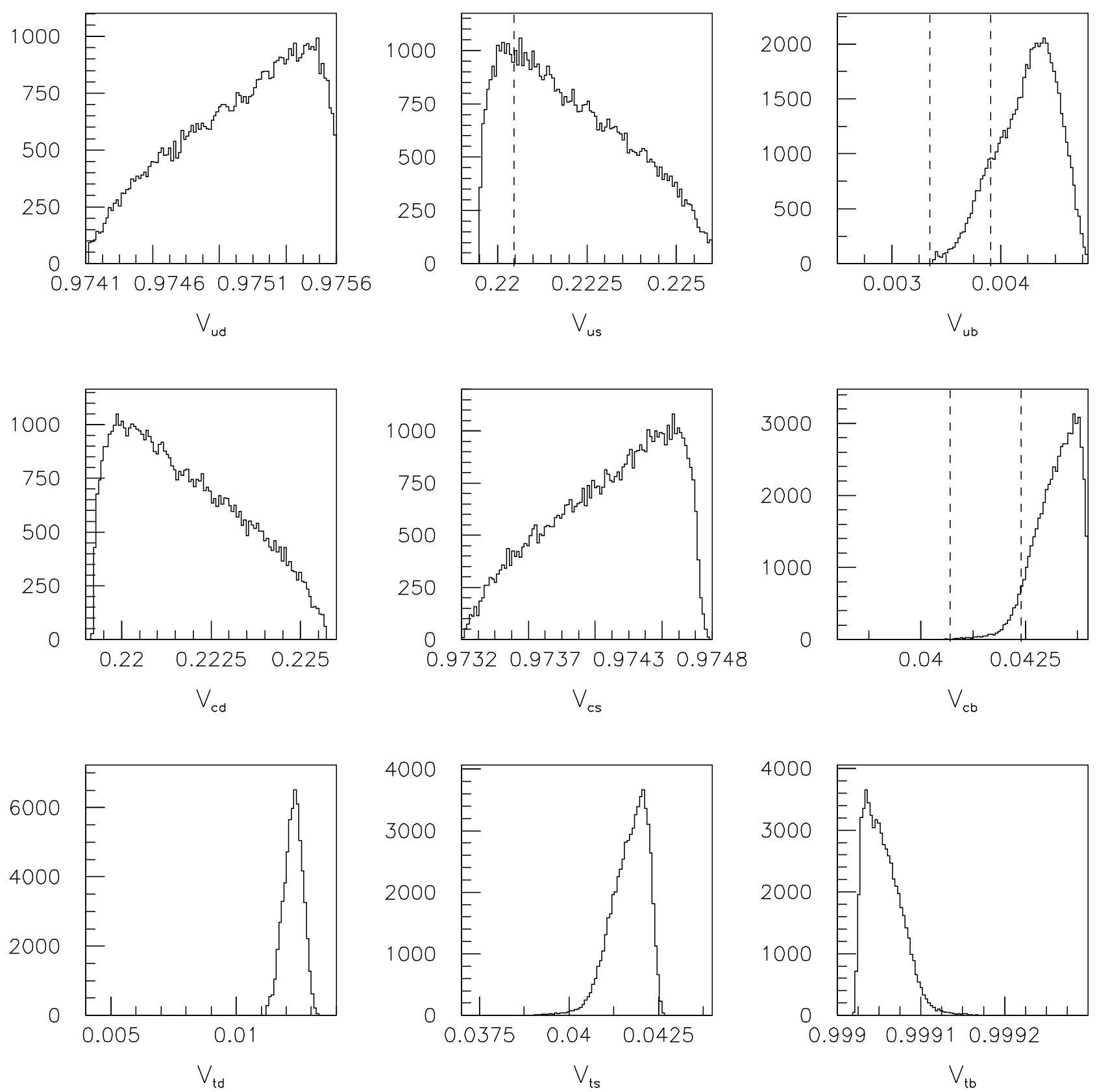

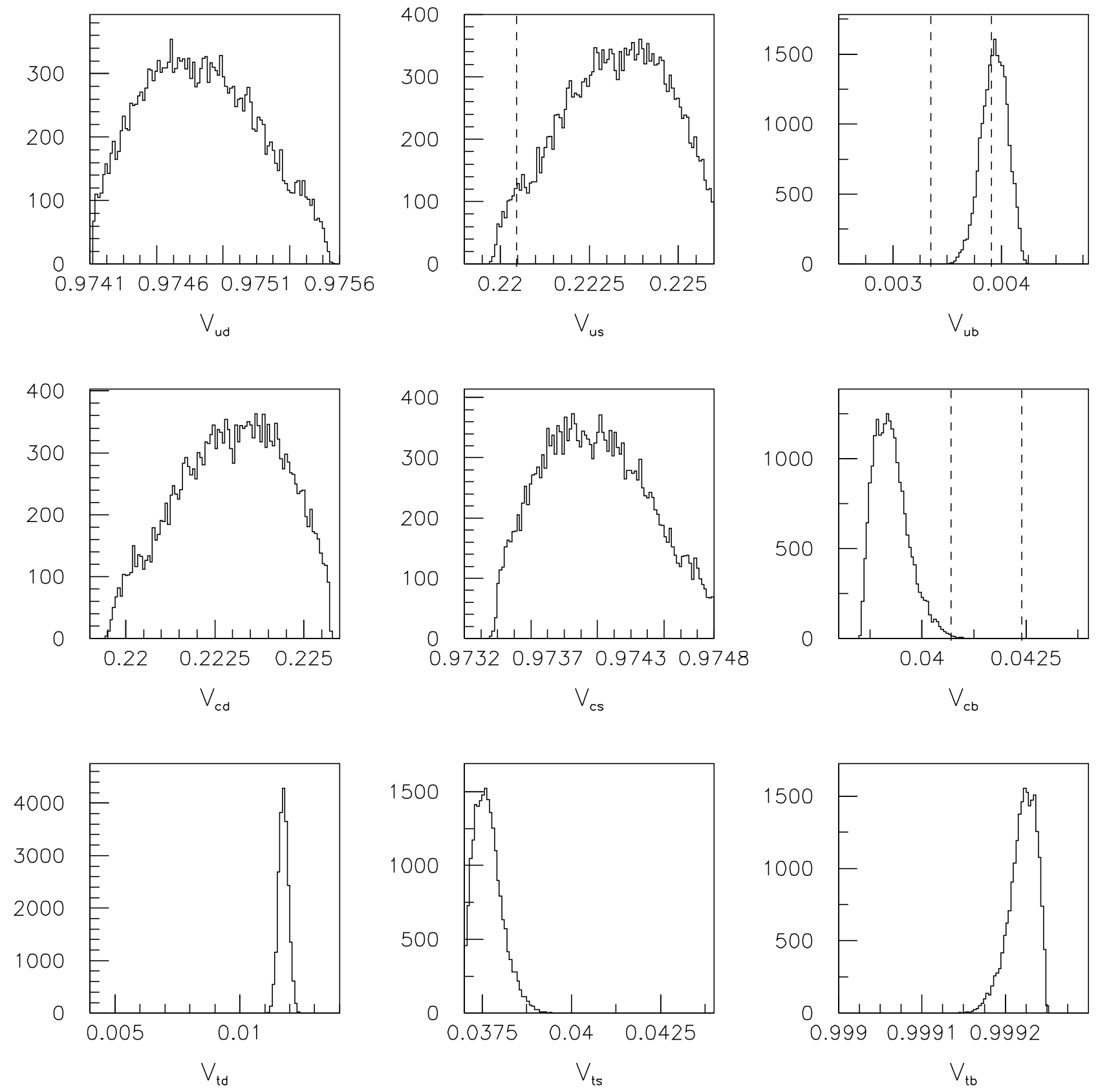

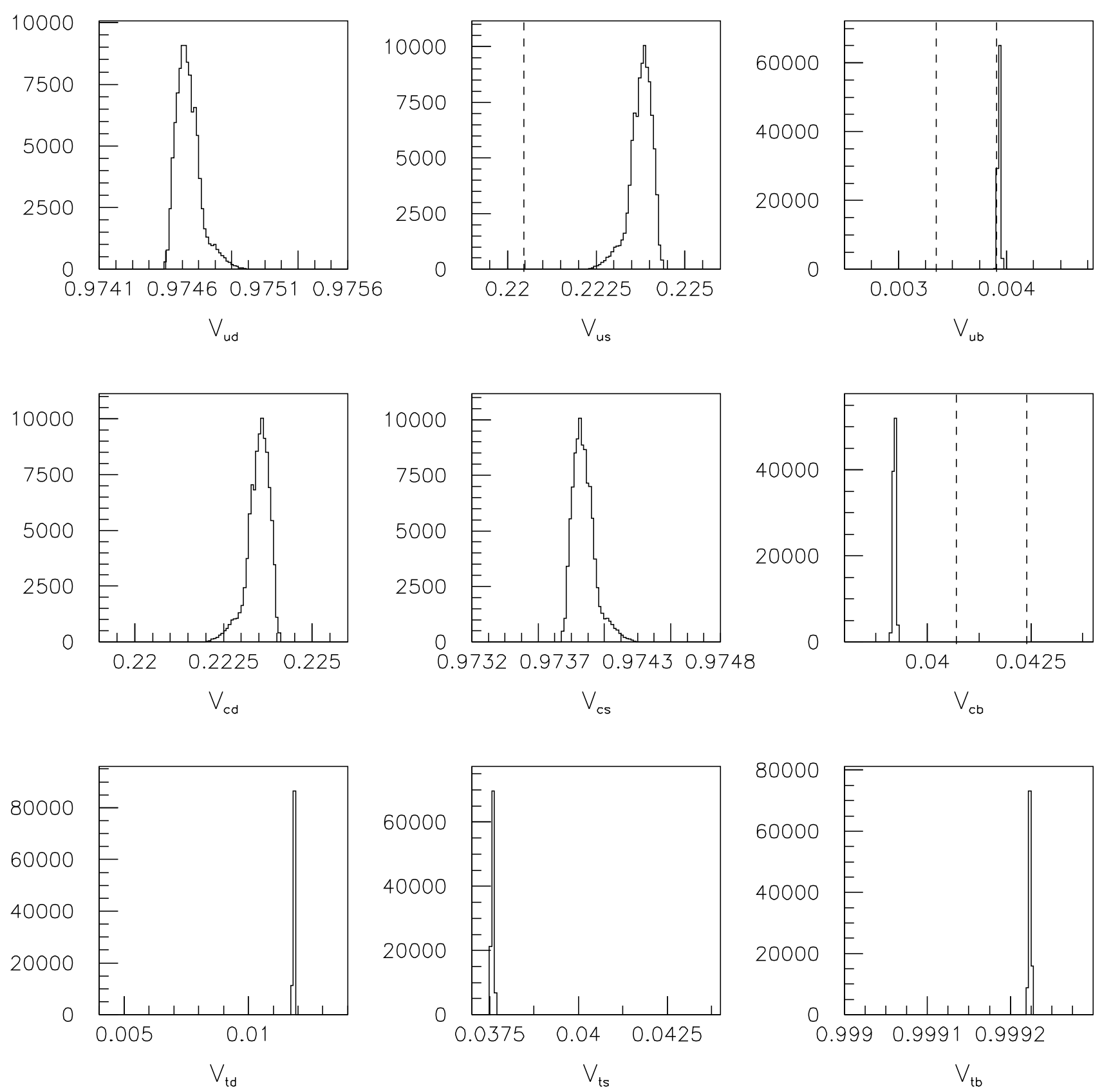
(a)

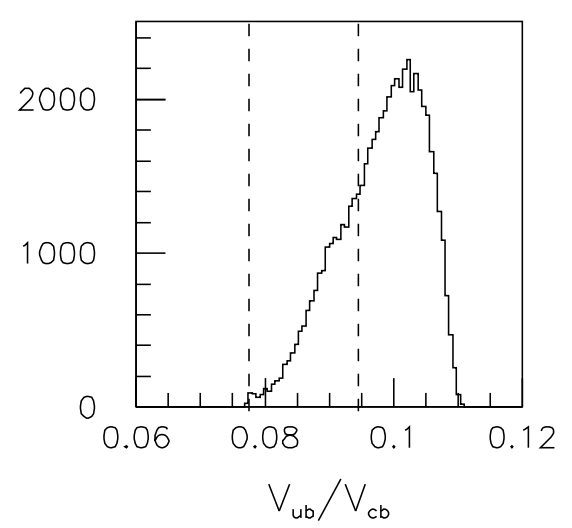

(b)

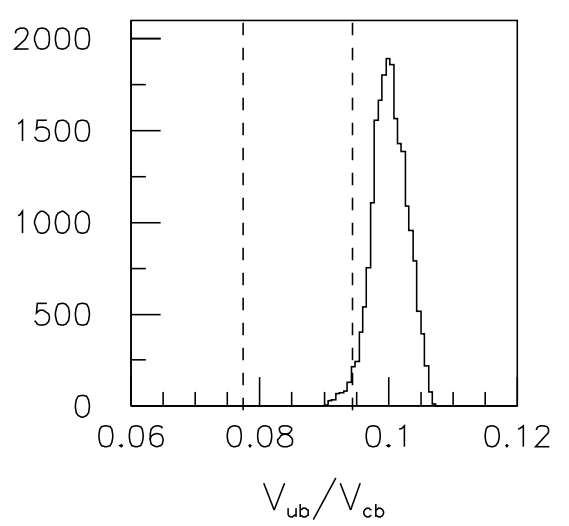

(c)

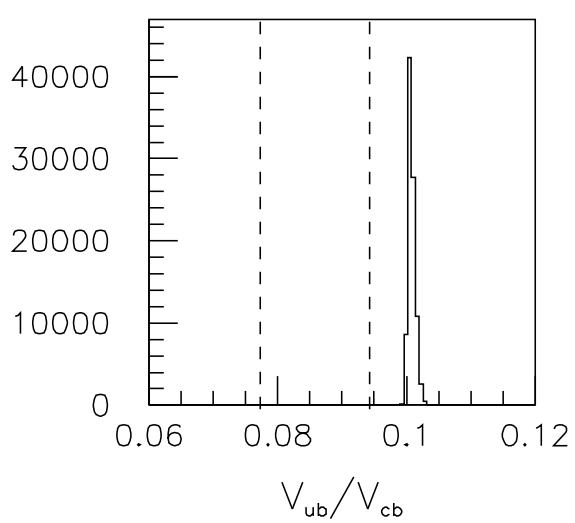


- - - Bayesian Method

Rfit Method
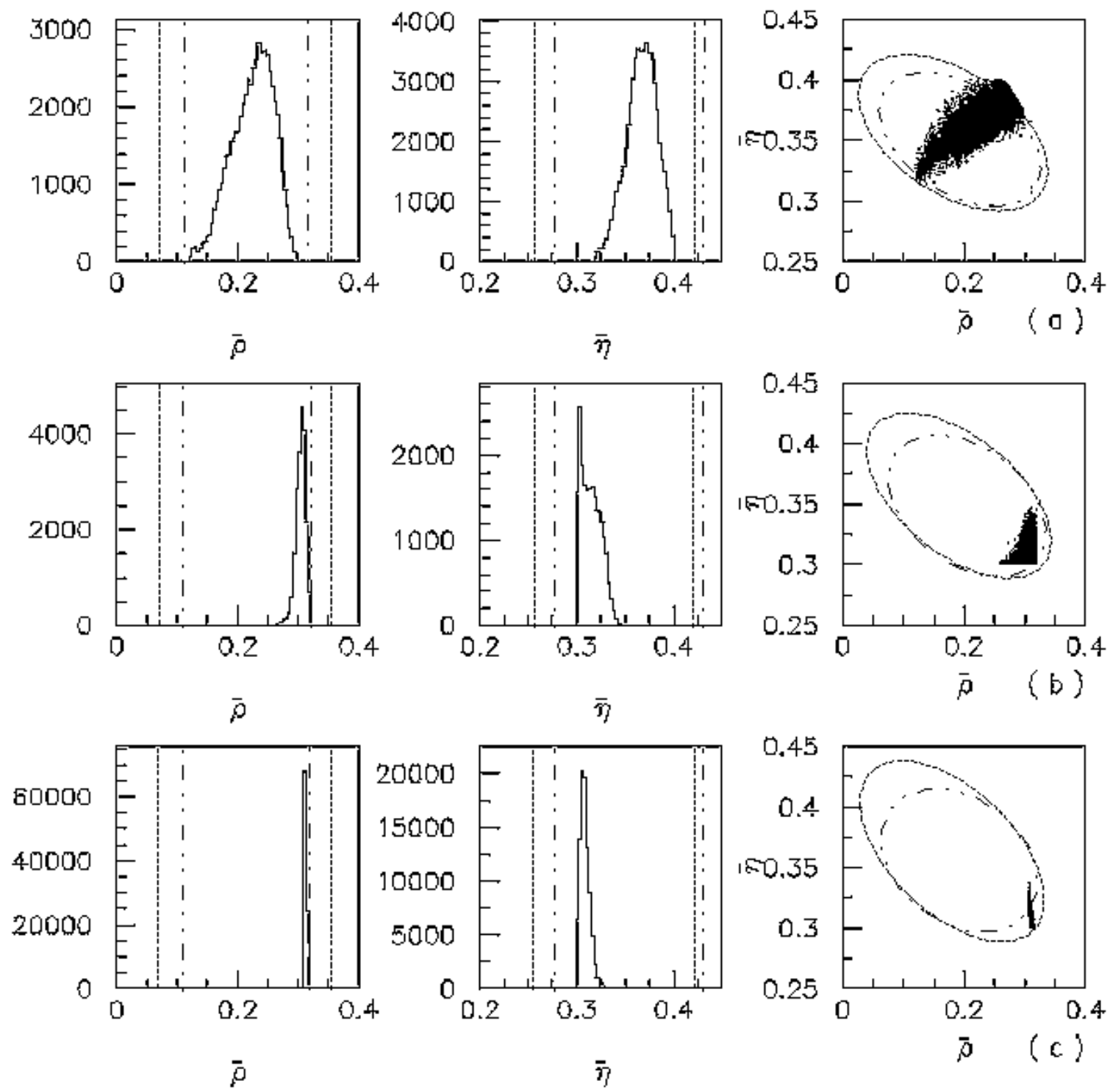

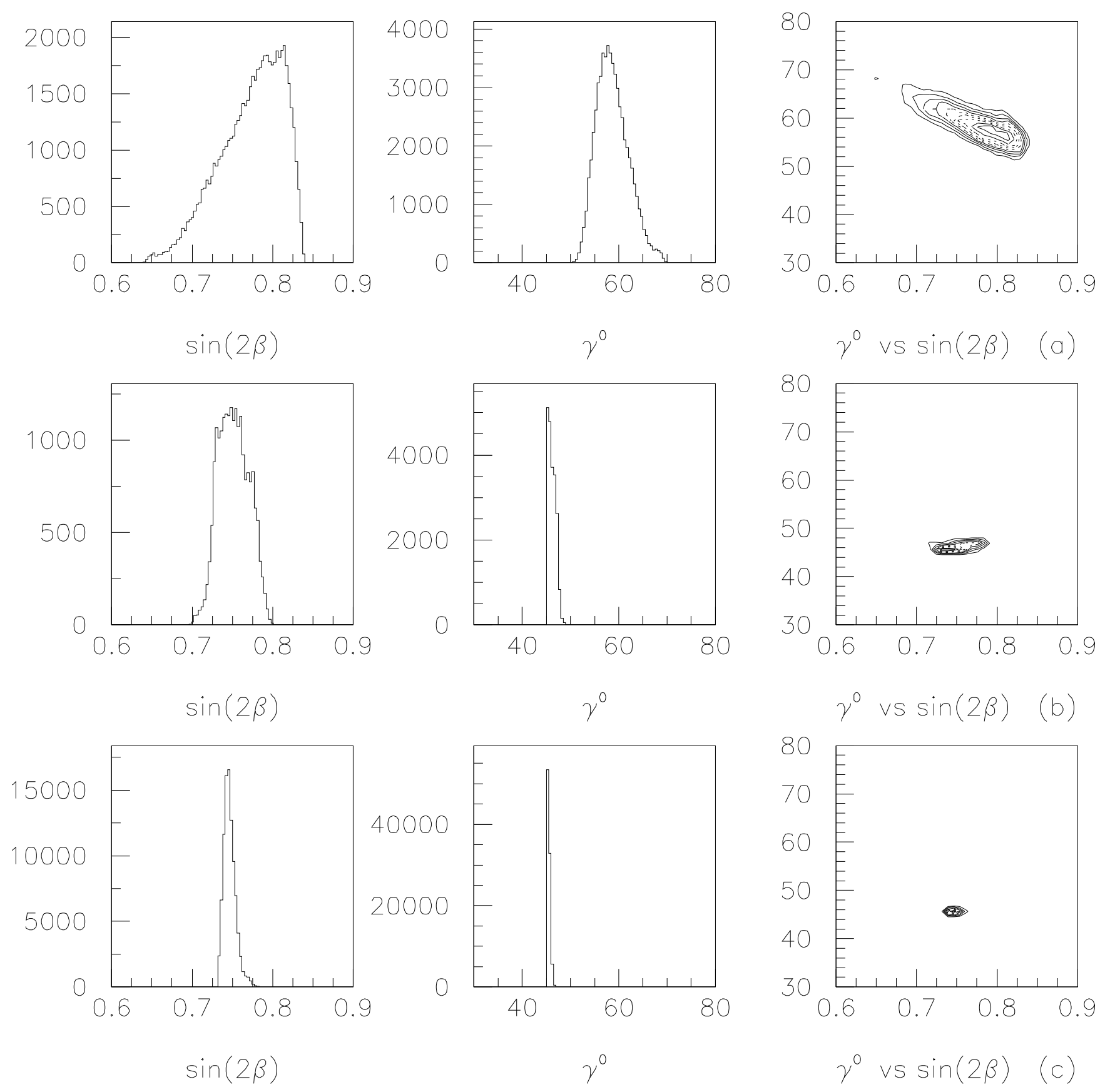

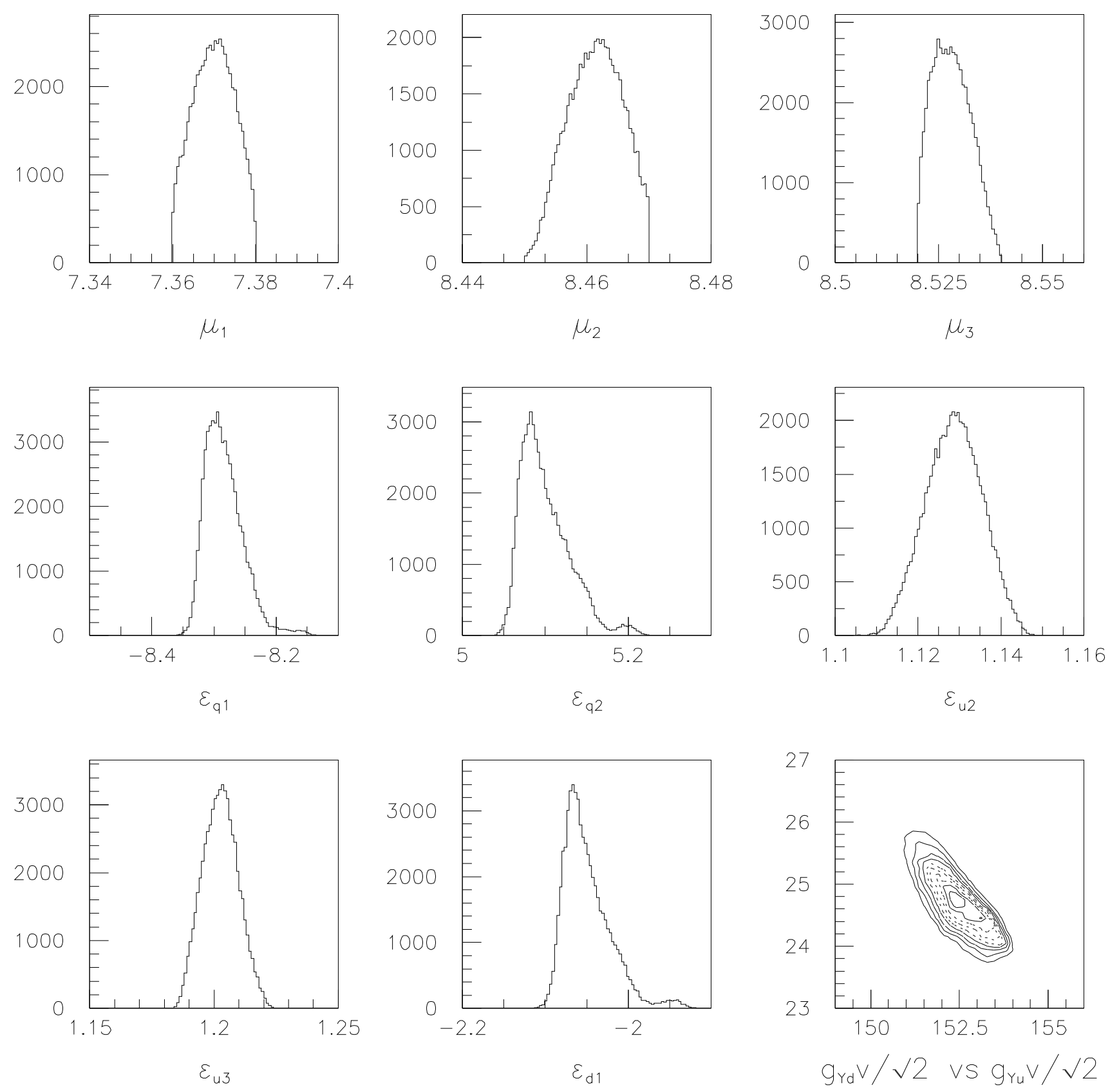

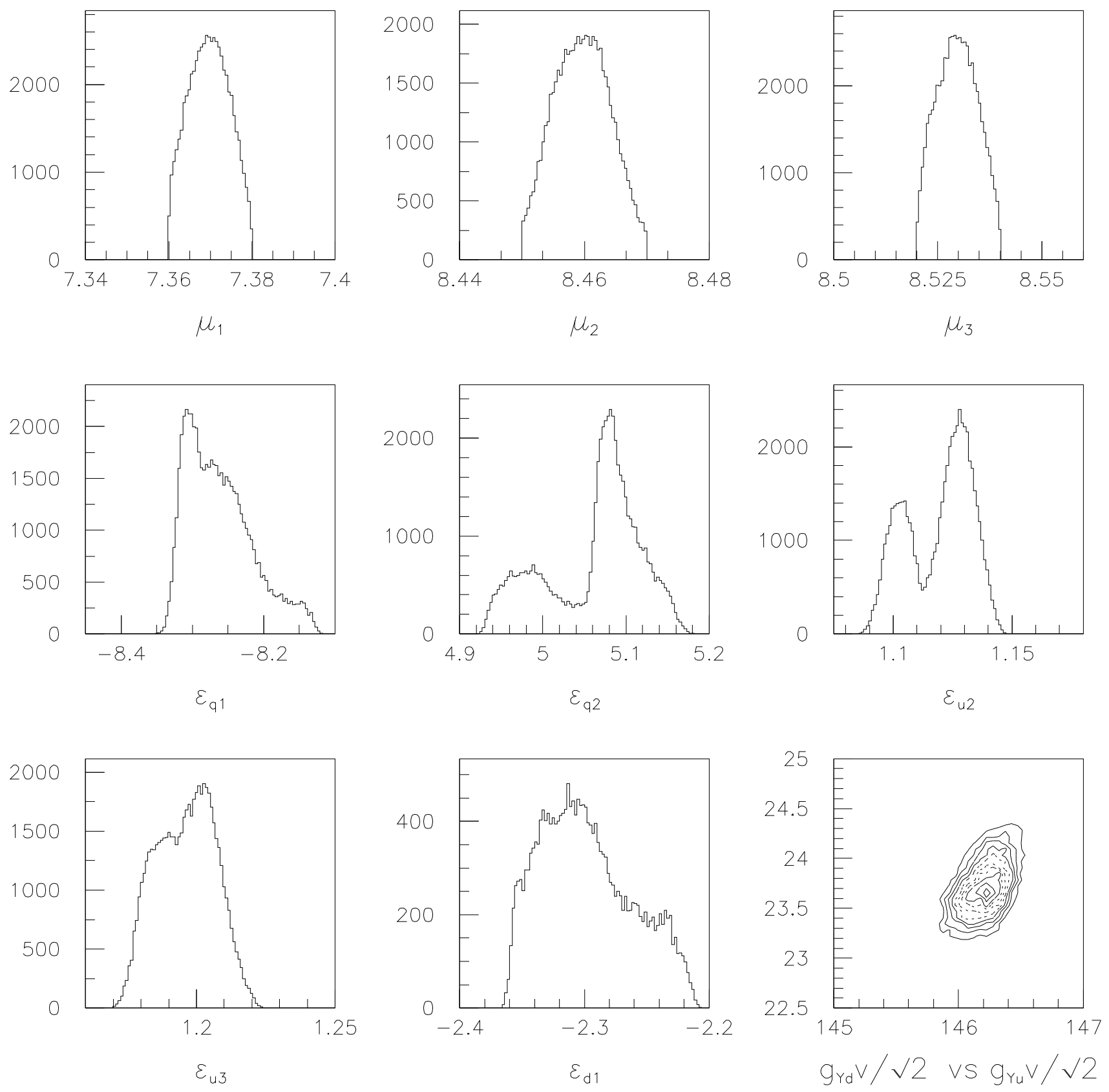

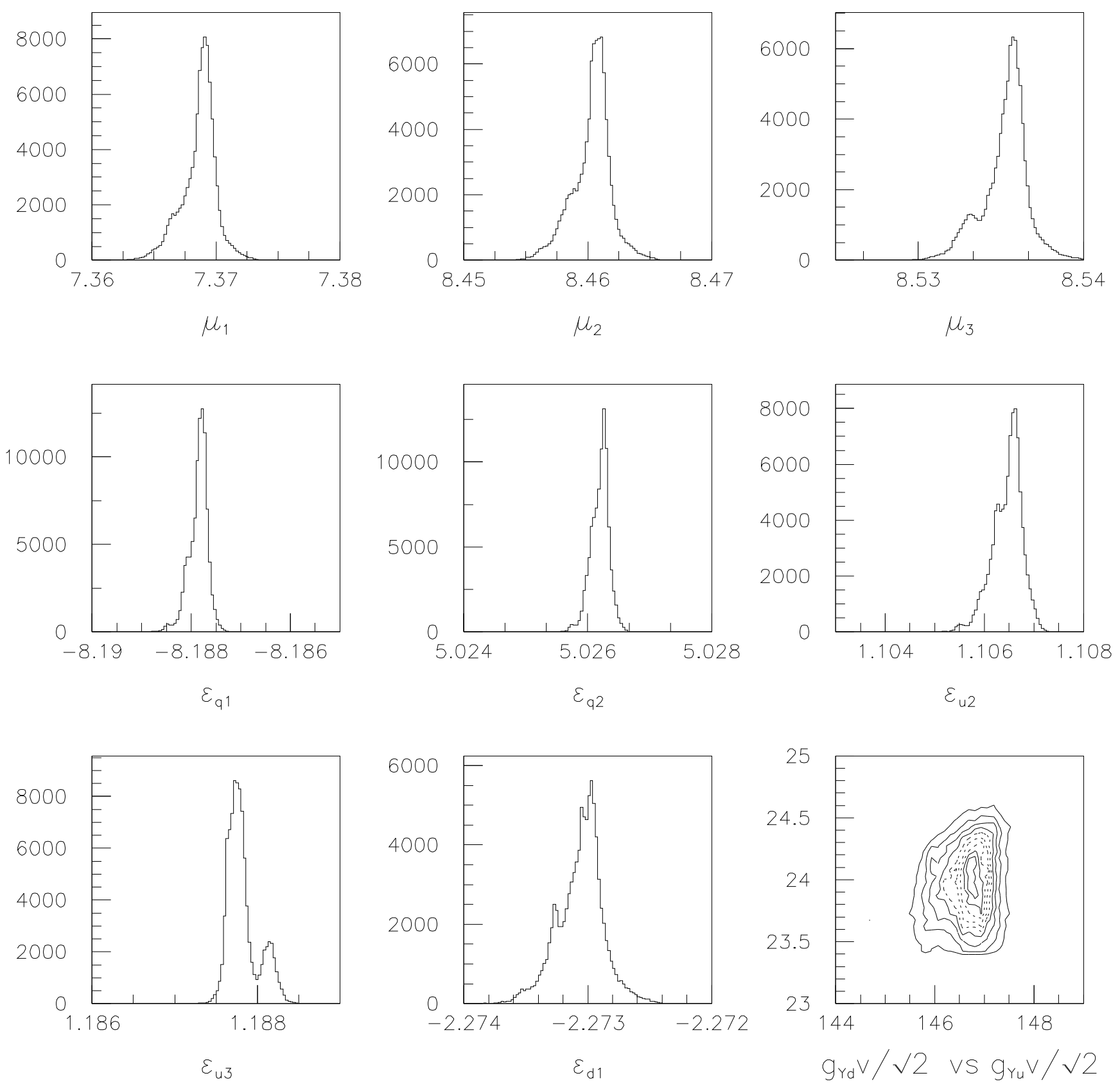


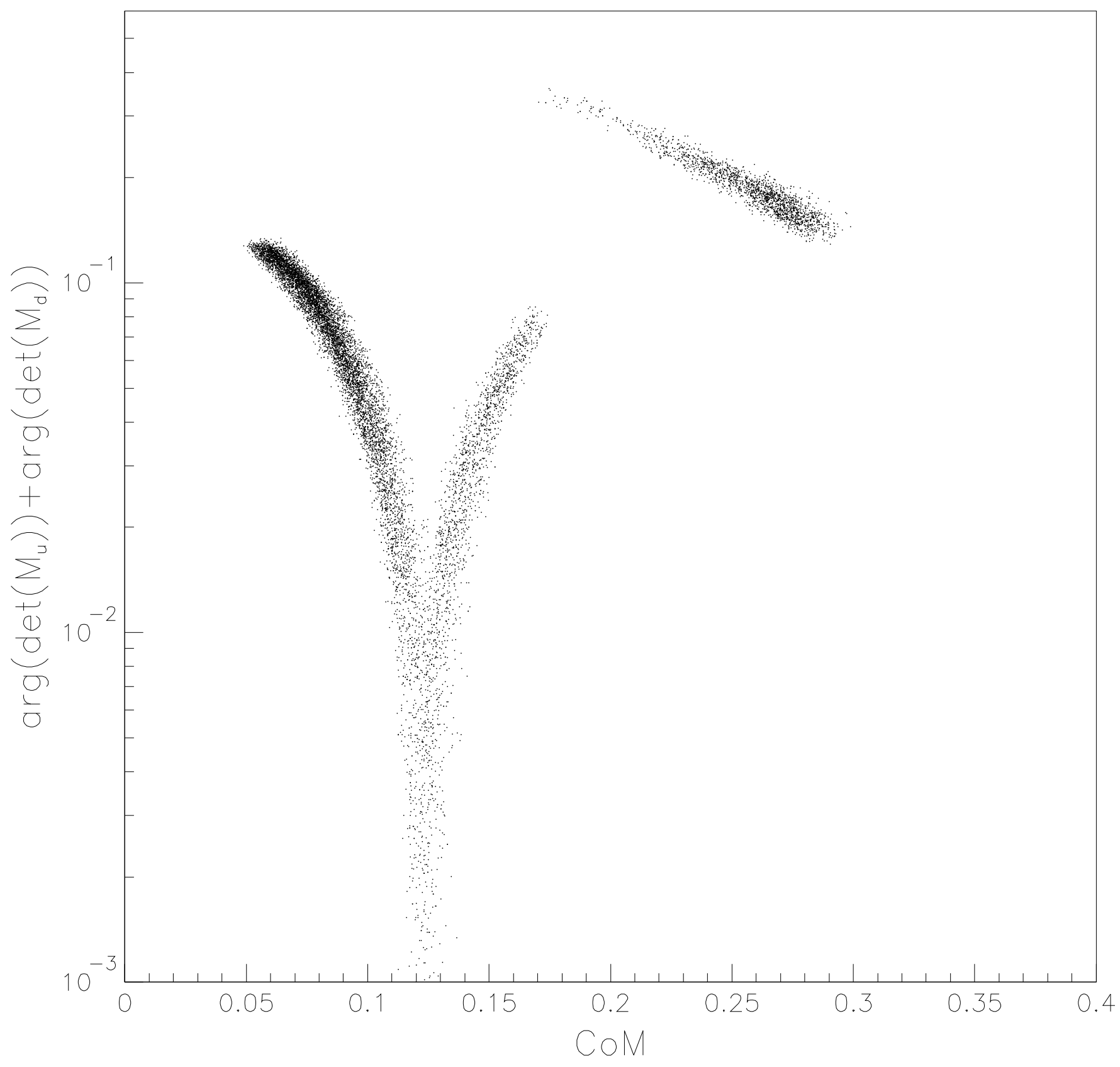




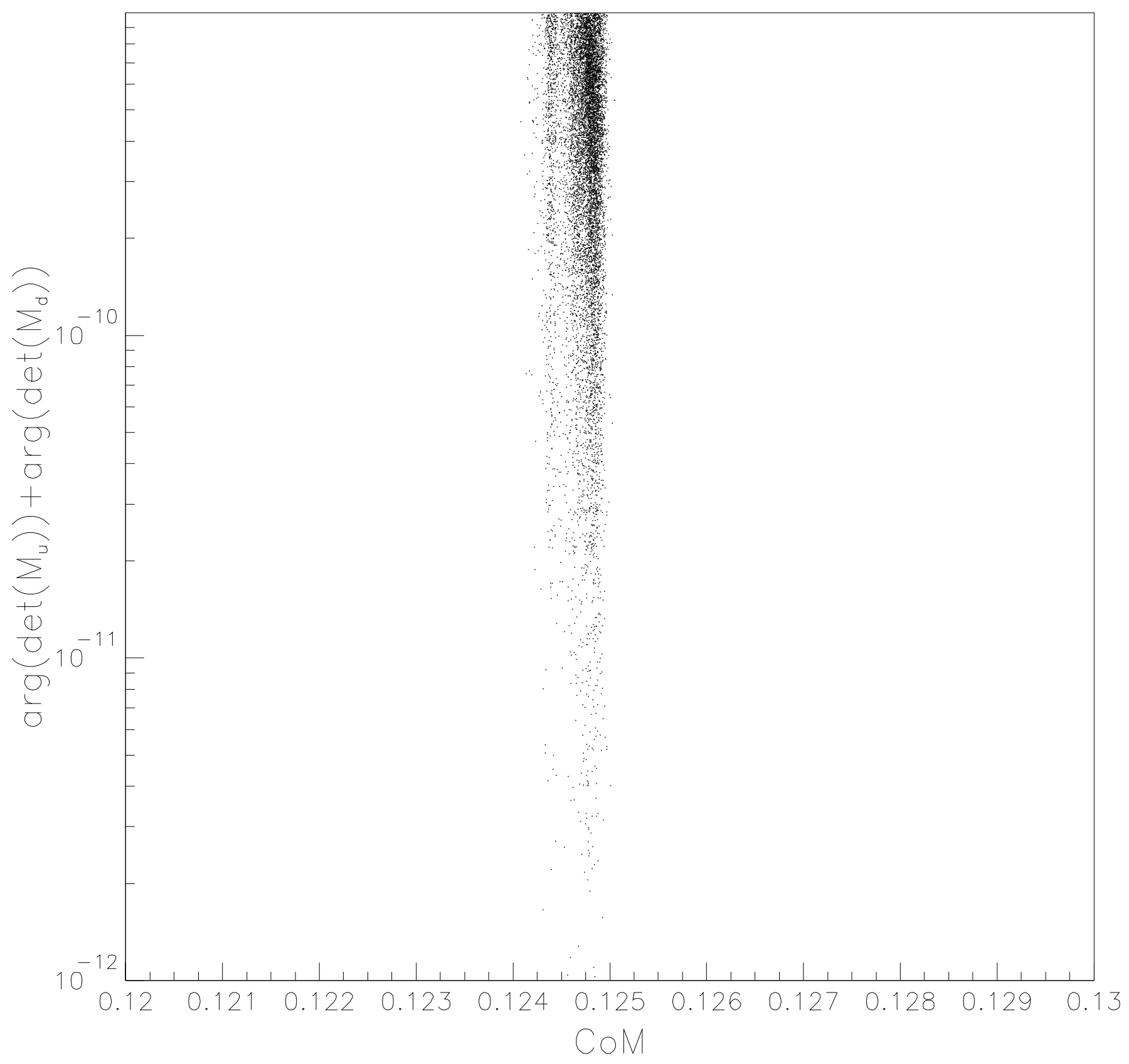

